Structural characterization of the reaction and substrate specificity mechanisms of pathogenic fungal acetyl-CoA synthetases.

Andrew J. Jezewski ${ }^{1}$, Katy M. Alden ${ }^{1}$, Taiwo E. Esan², Nicholas D. DeBouver ${ }^{3,5}$, Jan Abendroth ${ }^{3,5}$, Jameson C. Bullen ${ }^{3,5}$, Brandy M. Calhoun ${ }^{3,5}$, Kristy T. Potts ${ }^{3,4}$, Daniel M. Murante ${ }^{1}$, Timothy J. Hagen $^{2}$, David Fox ${ }^{3,4,5}$, and Damian J. Krysan*1,6

${ }^{1}$ Department of Pediatrics Carver College of Medicine, University of lowa, lowa City, IA 52242

${ }^{2}$ Department of Chemistry and Biochemistry, Northern Illinois University, DeKalb, IL 60115

3UCB Pharma., 7869 NE Day Road West, Bainbridge Island, WA 98110

${ }^{4}$ Beryllium Discovery Corp., 7869 NE Day Road West, Bainbridge Island, WA 98110

${ }^{5}$ Seattle Structural Genomics Center for Infectious Disease (SSGCID), Seattle, WA, 98109

${ }^{6}$ Microbiology/Immunology, Carver College of Medicine, University of lowa, lowa City, IA 52242

Running title: Substrate specificity of acetyl-CoA synthetases

Corresponding Author:

Damian J. Krysan

2040 Med Labs 25 S. Grand Avenue, Department of Pediatrics and Microbiology/lmmunology, Carver College of Medicine, University of lowa, lowa City lowa 52242, Phone: 319-335-3066, damian-krysan@uiowa.edu 


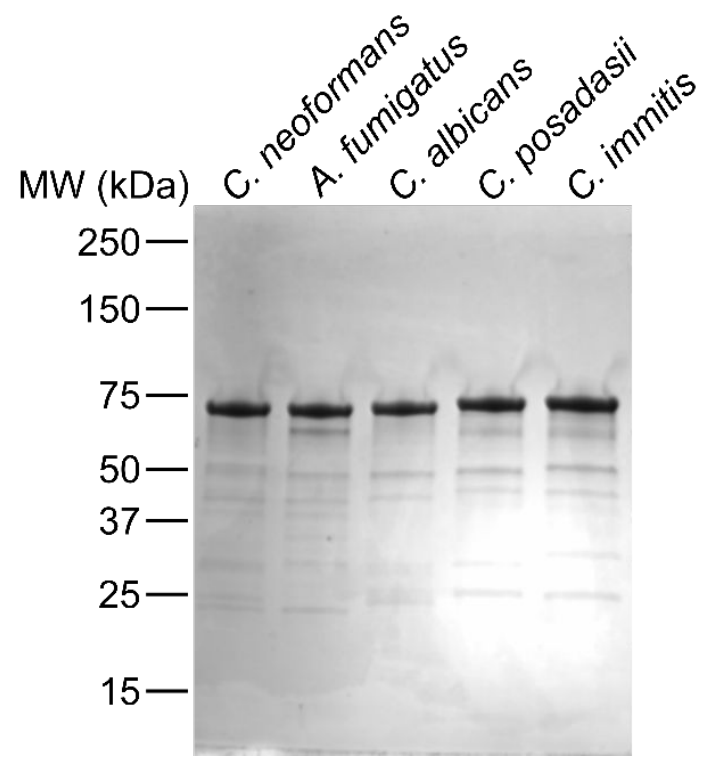

Supplemental Figure 1. Recombinantly expressed and purified Acs1 proteins. Coomassie gel represents 2 ug of loaded purified proteins with dominant bands representing $>95 \%$ purity. 
A

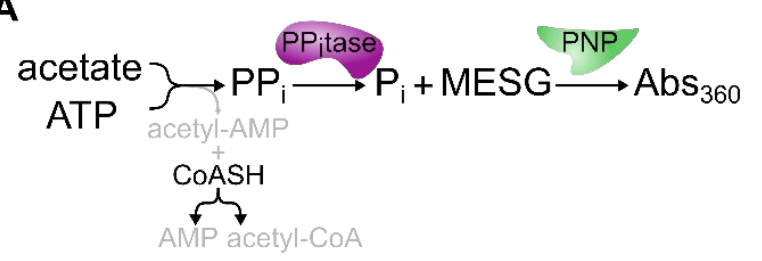

B

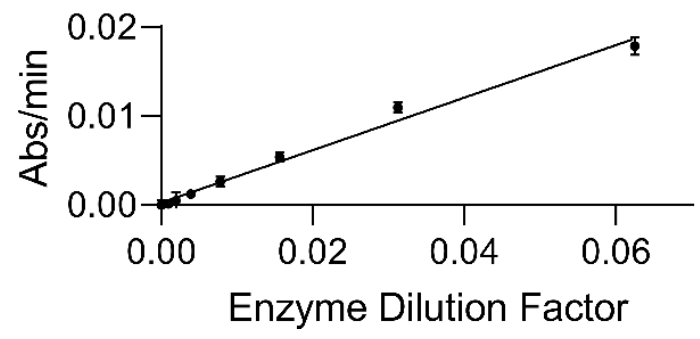

C

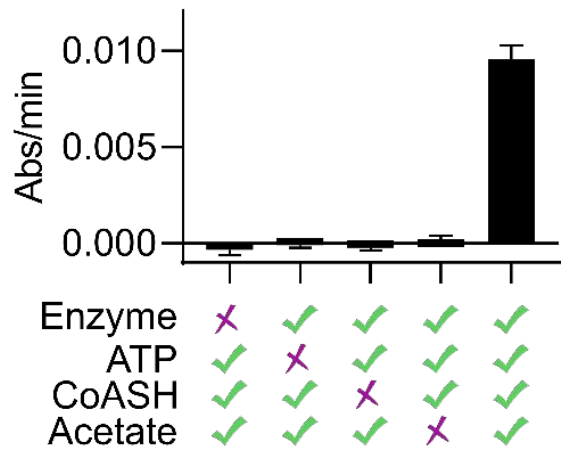

Supplemental Figure 2. ACS activity assay validation. (A) Schematic of ACS activity coupled to accessory enzyme detection of pyrophosphate (PPi) production. (B) Representative enzyme dilution curve to assure activity is measured within the linear range of enzyme content. (C) Detected activity is enzyme and substrate dependent. 


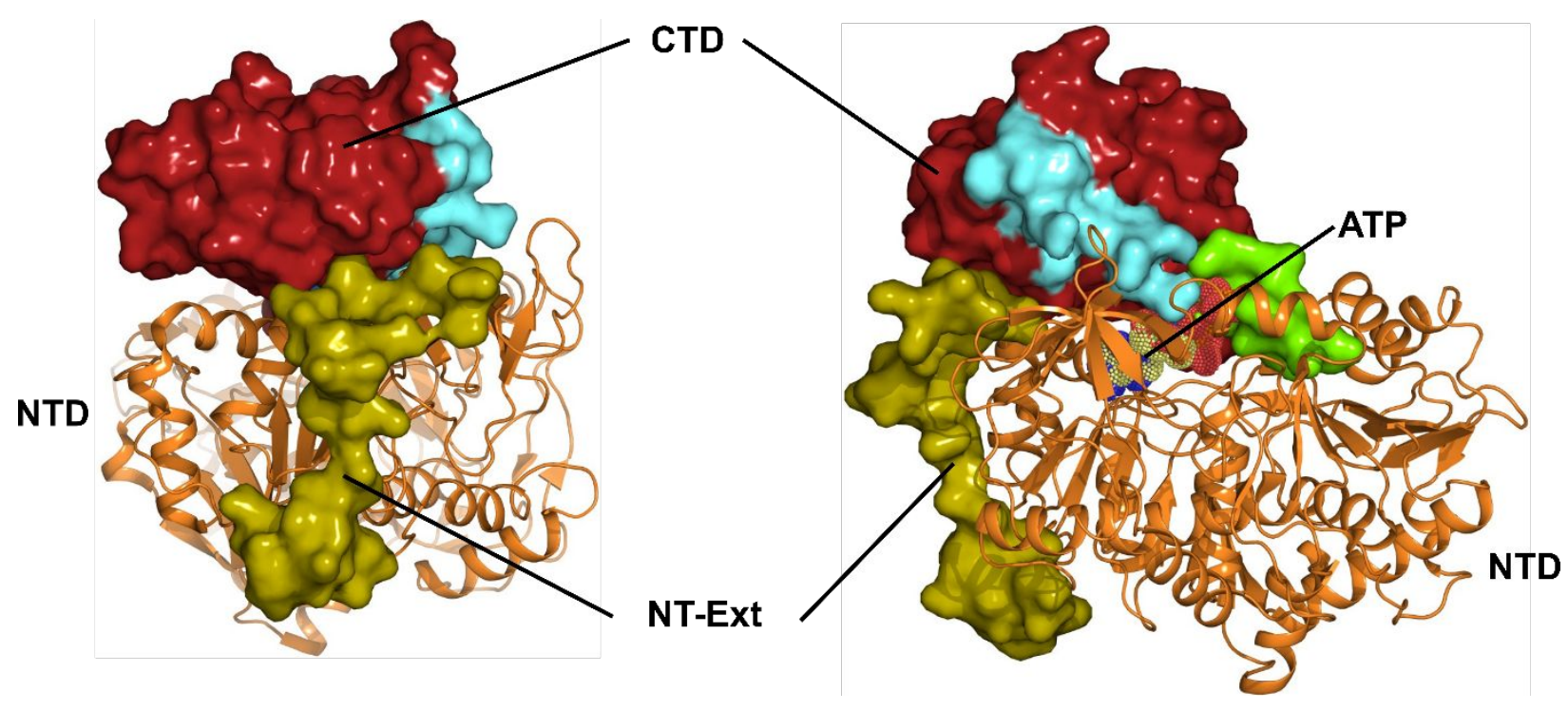

Supplementary Figure 3. N-terminal Extension (NT-Ext) from Cryptococcus neoformans Acs1. The ATP bound state (PDB 5K8F) is displayed with surfaces shown for NT-Ext (olive green), C-terminal domain (CTD, dark red), ATP binding loop (chartreuse) and hinge region (cyan). N-terminal domain (NTD) is shown in orange cartoon and ATP (yellow mesh). 

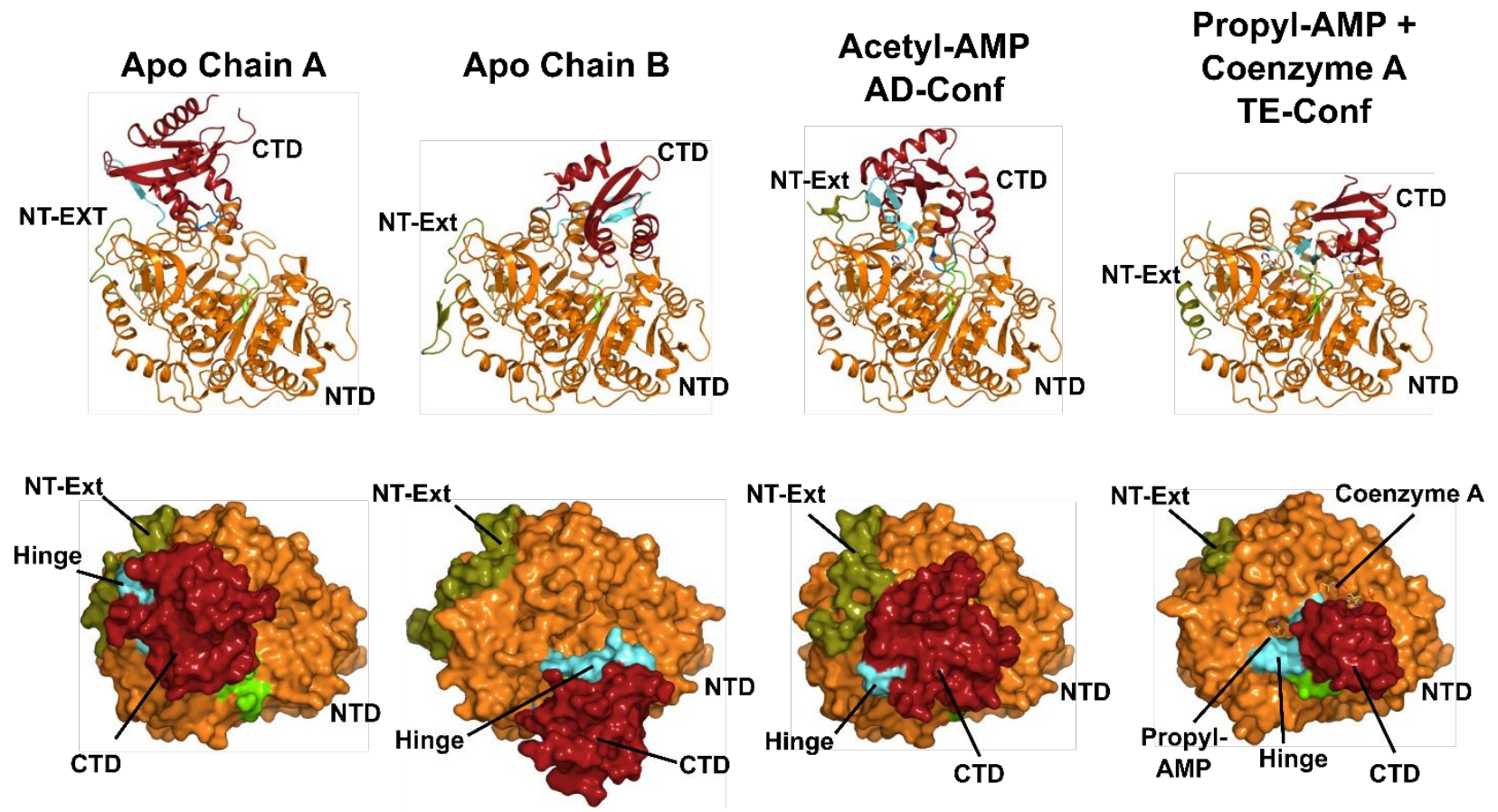

Supplemental Figure 4. Conformational orientations of the C-terminal Domain of Cryptococcus neoformans Acs1. (A-B) Apo (PDB 5PVP), (C) acetylating-conformation (ADconf) bound to Acetyl-AMP (PDB 74LG) and (D) thioesterification conformation (TE-conf) bound to Propyl-AMP and Coenzyme A (PDB 5K85). N-terminal domain (NTD, orange), C-terminal domain (CTD, dark red), N-terminal extension (NT-Ext, olive green), hinge (cyan), and ATP binding loop (Chartreuse). 

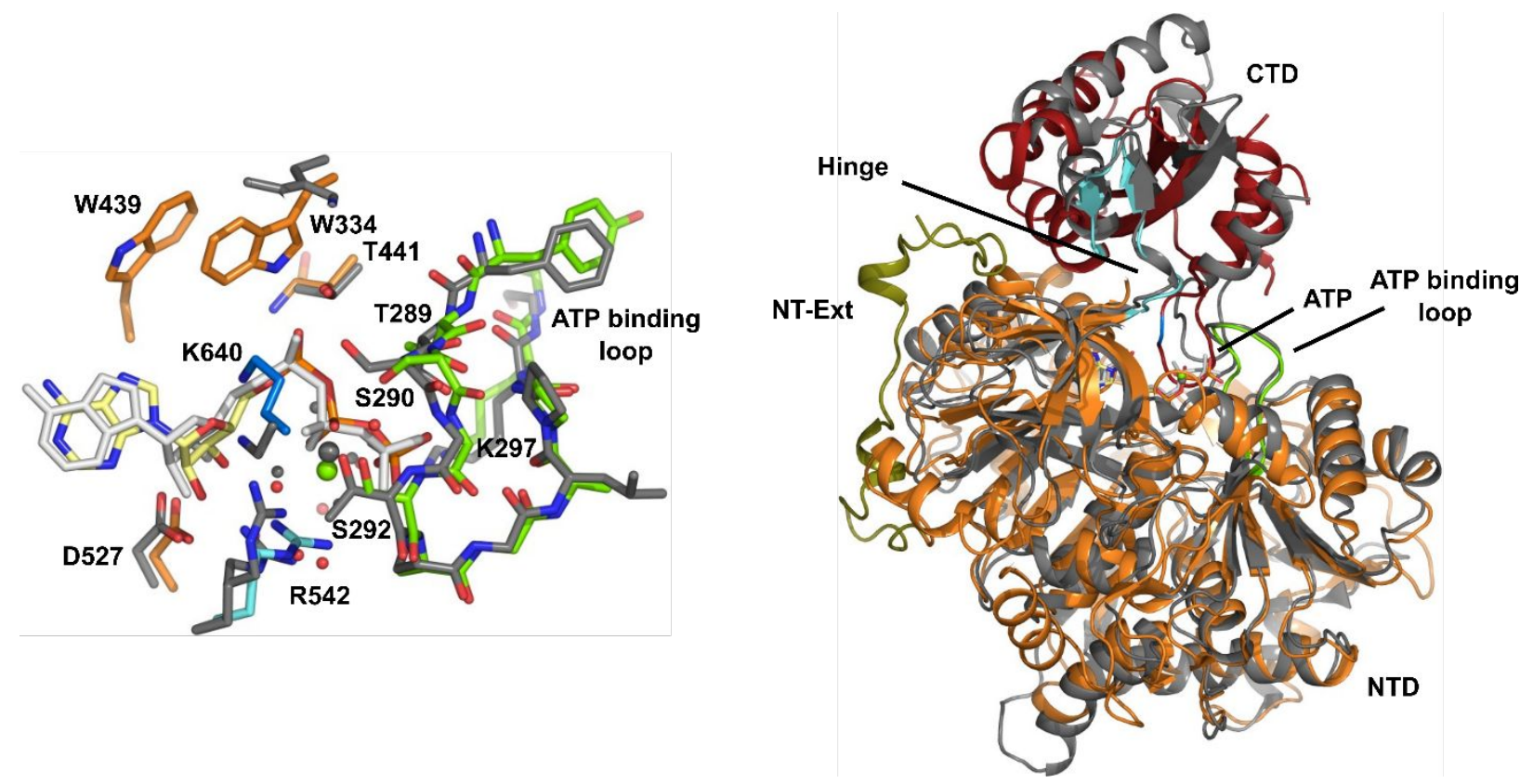

Supplemental Figure 5. Comparison of Human ACSM2A and Cryptococcus neoformans Acs1 bound to ATP and Magnesium. Human ACSM2A (PDB 3C5E) is shown in gray (cartoon) and light gray (compound). Cryptococcus neoformans Acs1 is shown in orange (N-terminal domain, NTD), dark red (C-terminal domain, CTD), olive green (N-terminal extension, NT-Ext), chartreuse (ATP binding loop), yellow (ATP), cyan (hinge), and blue (Active site Lys640). Right, overlay of $\mathrm{N}$-terminal domain in PyMol shown in cartoon with (left) active site shown in stick. Residue numbering shown for Cryptococcus neoformans Acs1. 
A

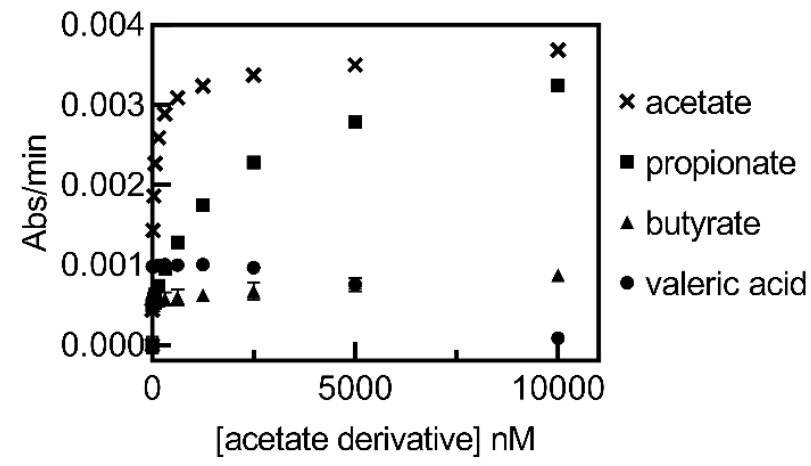

B

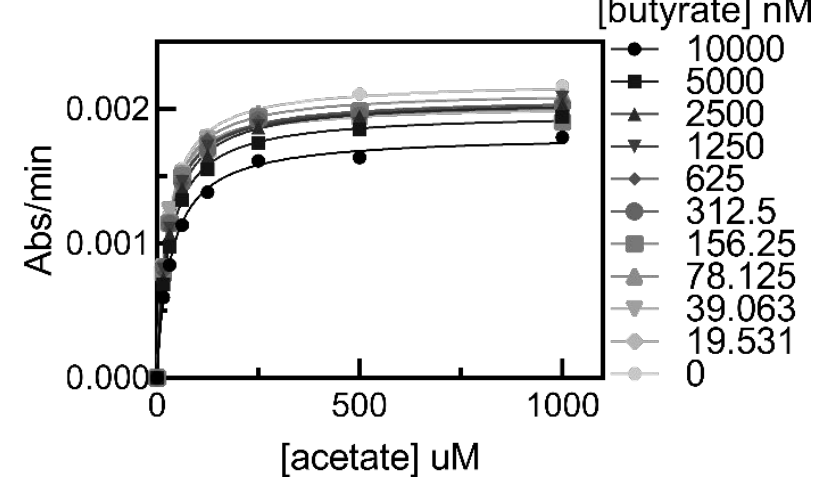

Supplemental Figure 6. Utilization of alternative acid substrates. (A) Activity of CnAcs1 against alternative acid substrates. (B) The non-preferred acid substrate butyrate does not inhibit acetate utilization. 
A

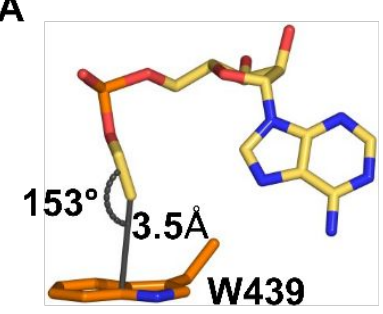

Ethyl-AMP

C

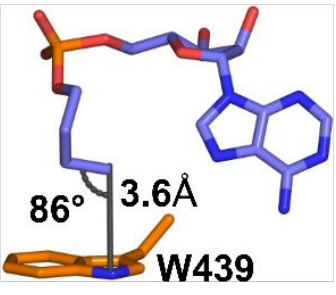

Butyl-AMP
B

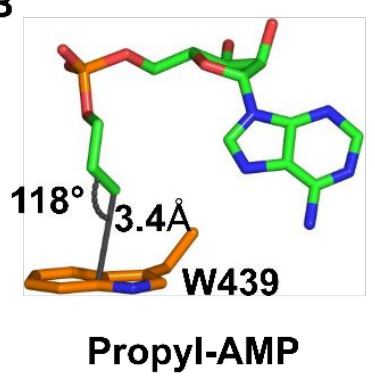

D

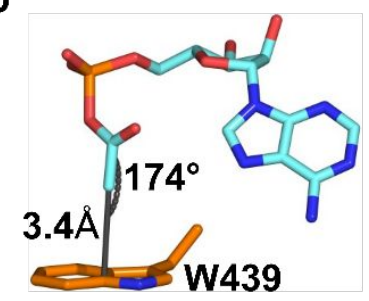

E

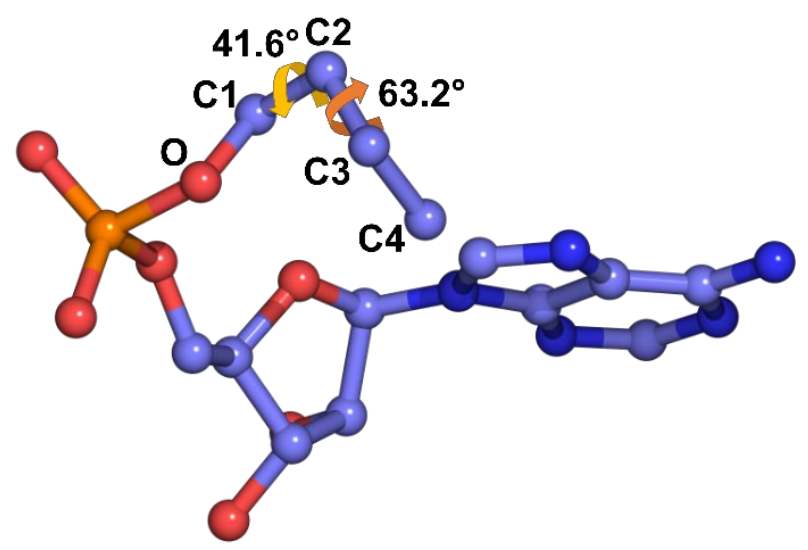

Butyl-AMP

Acetyl-AMP

Supplemental Figure 7. Angle and distances from terminal methyl to Trp439 sidechain in Cryptococcus neoformans AD-conformation. (A) Ethyl-AMP (PDB 7NKO, Chain A), (B) Propyl-AMP (PDB 5IFI, Chain A), (C) Butyl-AMP (PDB 7KNP, Chain A), and (D) Acetyl AMP (PDB 74LG, Chain A). Angles were measured from the two carbon atoms nearest Trp439 (central atom always as the terminal carbon) and nearest atom on Trp439 side-chain (Ethyl, propyl, and acetyl = C $\varepsilon 2 ;$ Butyl $=\mathrm{N} \varepsilon 1$ ). Distances measured from terminal carbon atom and center of Trp439 ring (Ethyl, propyl and acetyl = center of both rings; Butyl = 5-membered ring only). (E) Dihedral angles measured for butyl AMP (PDB 7KNP) for O-C1-C2-C3 (yellow arrow) and C1-C2-C3-C4 (orange arrow). 


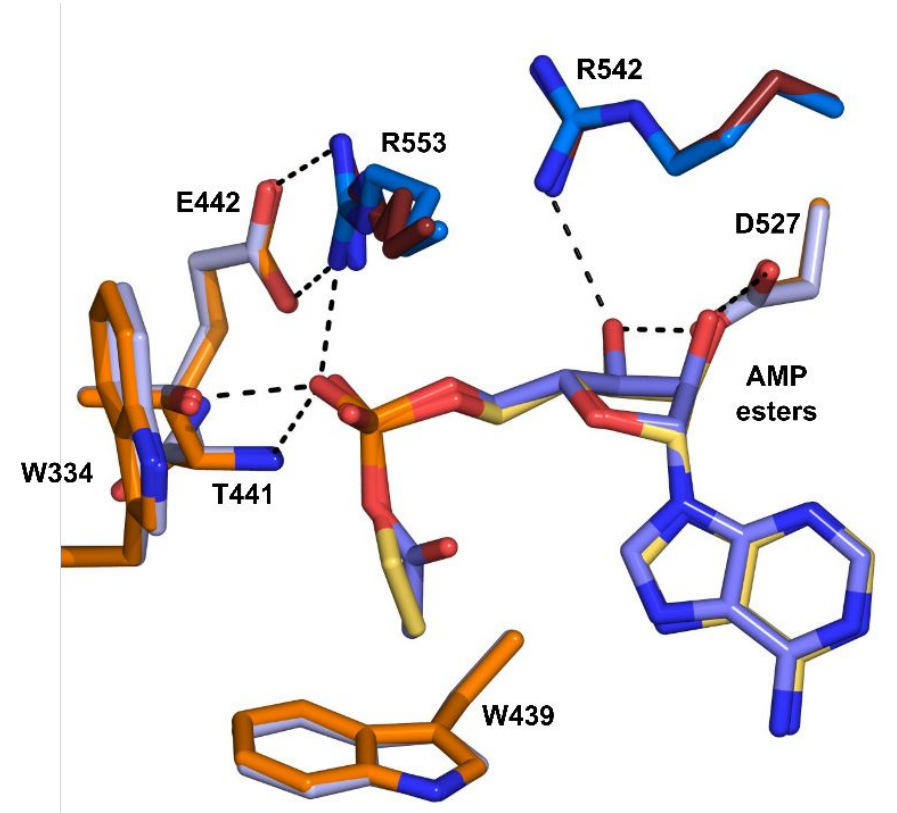

Supplemental Figure 8. Comparison of ethyl-AMP and acetyl-AMP bound to Cryptococcus neoformans Acs1. Overlay of ethyl-AMP (PDB 7KNO, compound - yellow, protein - orange) and acetyl-AMP (PDB 74LG, compound - blue, protein - light blue). 


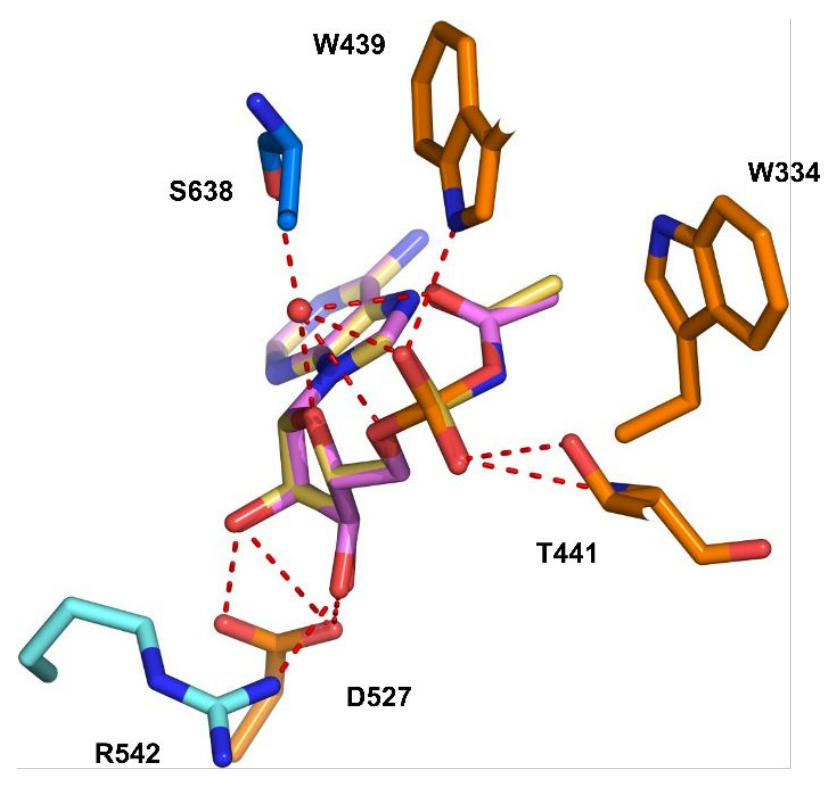

Cryptococcus neoformans

(AD-conf, Acetyl-AMS vs Acetyl-AMP

Supplemental Figure 9. Overlay of Acetyl-AMP and Acetyl-AMS crystal structures. AcetylAMP (PDB 74LG) and Acetyl-AMS (PDB 5U29) structures were overlaid in PyMol. Compounds are shown in stick representation with Acetyl-AMP in yellow and Acetyl-AMS in pink. Acetyl-AMS residues are shown surrounding the compound. 

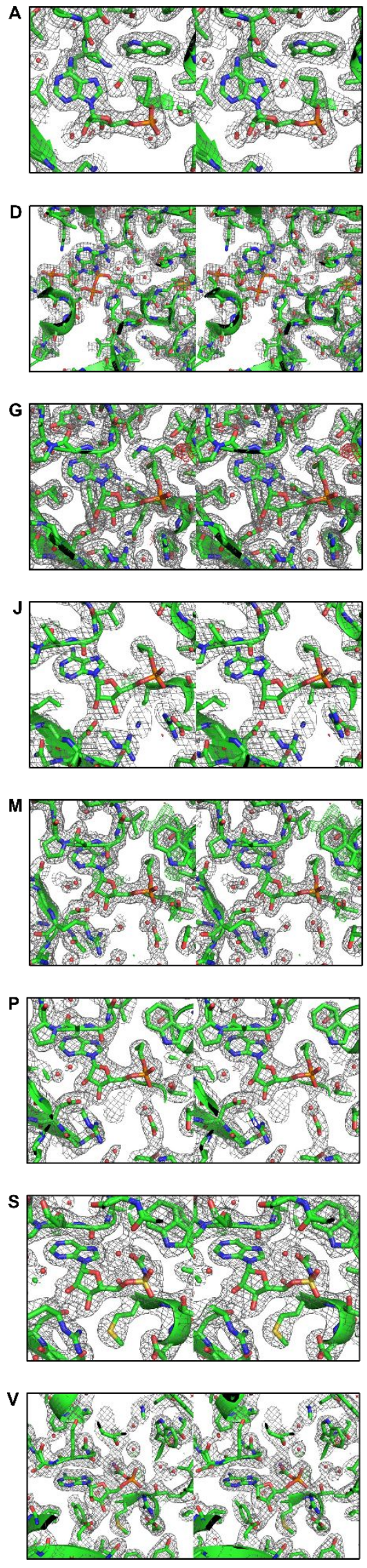
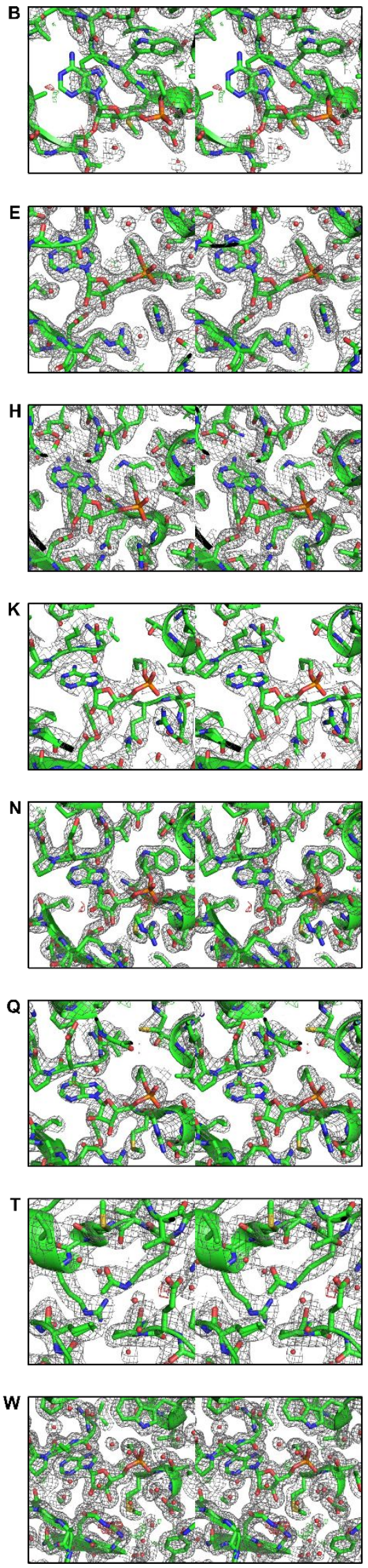
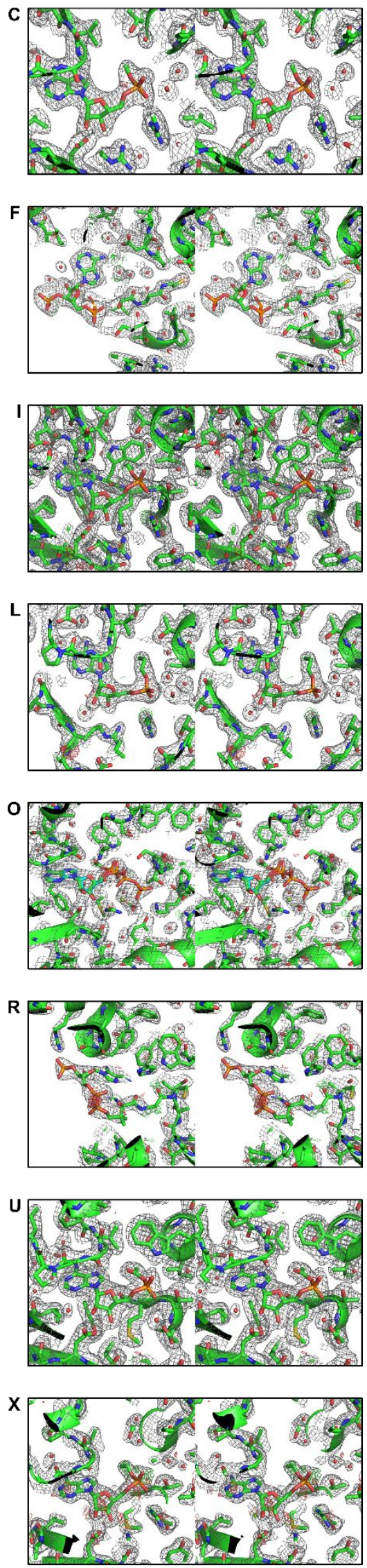
Supplemental Figure 10. Stereo images of substrate binding pockets for fungal Acs1 crystal structures. Cryptococcus neoformans + butyl AMP (PDB 7KNP), (A) Chain A (AD conformation) and (B) Chain C (TE conformation). Coccidioides immitis + methyl AMP + coenzyme A (PDB 7L3Q), (C) Chain A, methyl AMP (TE conformation), and (D) Chain A, coenzyme A (TE conformation). Coccidioides immitis + ethyl AMP + coenzyme A (PDB 7KVY), (E) Chain A, ethyl AMP (TE conformation), and (F) Chain A, coenzyme A (TE conformation). Coccidioides immitis + propyl AMP (PDB 7KQ6), (G) Chain A (TE conformation). Coccidioides immitis + ethyl AMP (PDB 7KQZ), (H) Chain A (TE conformation). Coccidioides immitis + methyl AMP (PDB 7L3P), (I) Chain A (TE conformation). Aspergillus fumigatus + propyl AMP (PDB 7KDN), (J) Chain A (TE conformation). Candida albicans + propyl AMP (PDB 7KDS), (K) Chain A (TE conformation). Coccidioides posadasii + propyl AMP (PDB 7KCP), (L) Chain A (TE conformation). Cryptococcus neoformans + propyl AMP (PDB 5IFI), M) Chain A (AD conformation) and N) Chain C (TE conformation). Cryptococcus neoformans + ATP/acetyl AMP (PDB 5K8F), O) Chain A (AD conformation). Cryptococcus neoformans + propyl AMP + coenzyme A (PDB 5K85), P) Chain A (AD conformation) and Q) Chain C, propyl AMP (TE conformation), and R) Chain C, coenzyme A (TE conformation). Cryptococcus neoformans + acetyl AMS (PDB 5U29), S) Chain A (AD conformation). Cryptococcus neoformans + acetyl lysine (PDB 5VPV), T) Chain A. Cryptococcus neoformans + acetyl AMP (PDB 74LG), U) Chain A (AD conformation) and V) Chain C (TE conformation). Cryptococcus neoformans + ethyl AMP (PDB 7KNO), W) Chain A (AD conformation) and X) Chain C (TE conformation). 2Fo-Fc maps contoured at $1.0 \sigma$ (grey), Fo-Fc maps contoured at $+3.0 /-3.0$ (green/red). Stereo images generated in PyMol. 


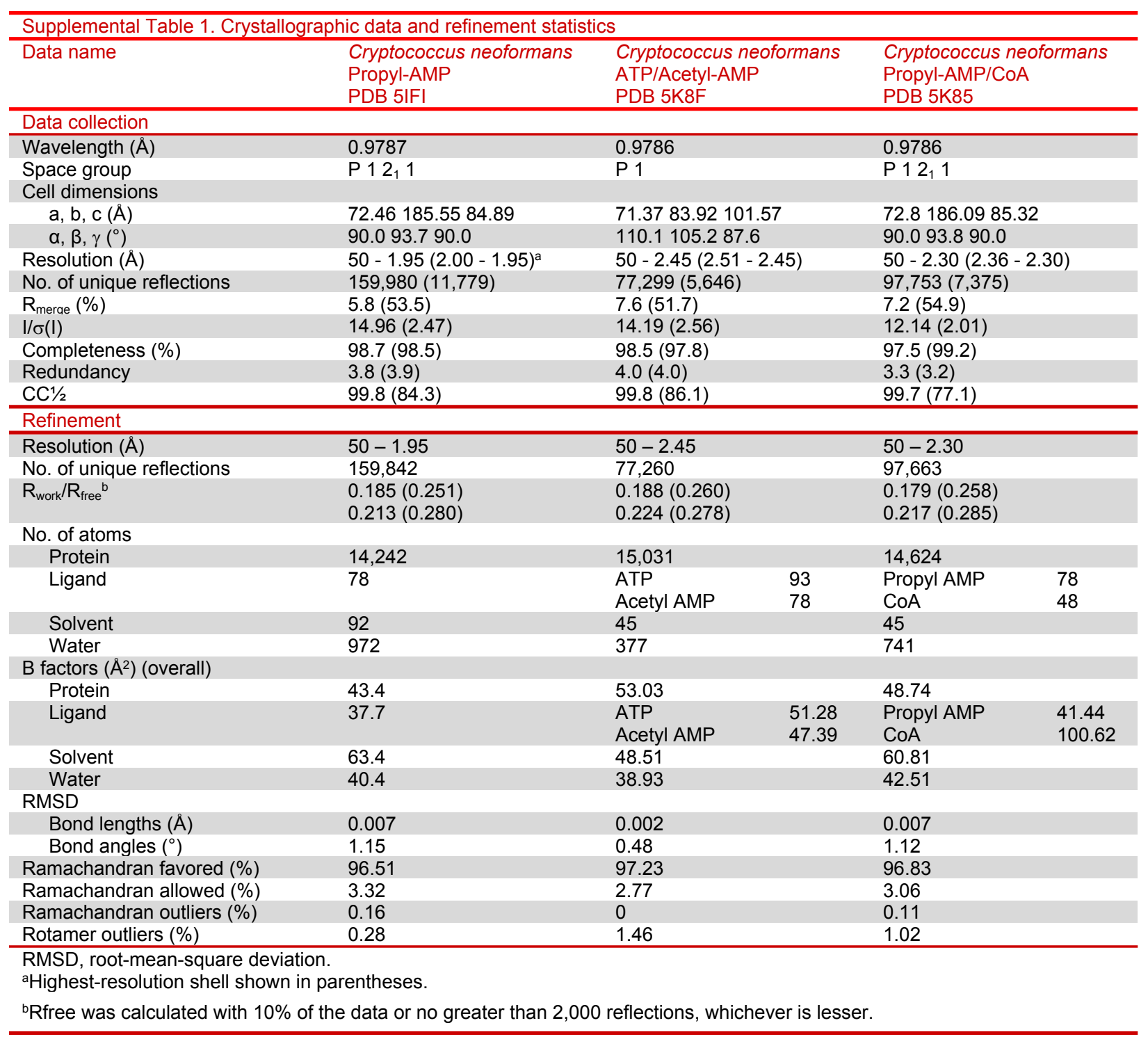




\begin{tabular}{|c|c|c|c|}
\hline Data name & $\begin{array}{l}\text { Cryptococcus neoformans } \\
\text { Acetyl-AMS } \\
\text { PDB 5U29 }\end{array}$ & $\begin{array}{l}\text { Cryptococcus neoformans } \\
\text { Apo } \\
\text { PDB 5VPV }\end{array}$ & $\begin{array}{l}\text { Cryptococcus neoformans } \\
\text { Acetyl-AMP } \\
\text { PDB 74LG }\end{array}$ \\
\hline \multicolumn{4}{|l|}{ Data collection } \\
\hline Wavelength $(\AA)$ & 0.9786 & 0.9787 & 0.9787 \\
\hline Space group & P 1 & $\mathrm{P} 4{ }_{1} 2_{1} 2$ & P 1211 \\
\hline \multicolumn{4}{|l|}{ Cell dimensions } \\
\hline$a, b, c(\AA)$ & 71.2883 .78101 .6 & 176.98176 .98159 .92 & 72.39185 .7584 .88 \\
\hline$\alpha, \beta, \gamma\left({ }^{\circ}\right)$ & 110.3105 .887 .8 & 90.090 .090 .0 & 90.094 .090 .0 \\
\hline Resolution $(\AA)$ & $50-2.50(2.56-2.50)$ & $50-2.60(2.67-2.60)$ & $50-2.20(2.26-2.20)$ \\
\hline No. of unique reflections & $72,292(5,310)$ & $78,331(5,723)$ & $110,867(8,124)$ \\
\hline $\mathrm{R}_{\text {merge }}(\%)$ & $7.3(58.8)$ & $16.0(52.1)$ & $8.0(62.5)$ \\
\hline $\mathrm{I} / \sigma(\mathrm{I})$ & $14.69(2.64)$ & $12.18(5.02)$ & $12.10(2.25)$ \\
\hline Completeness (\%) & $98.5(97.8)$ & $100.0(100.0)$ & $98.1(97.3)$ \\
\hline Redundancy & $3.9(4.0)$ & $12.4(12.7)$ & $4.8(4.8)$ \\
\hline $\mathrm{CC}^{1} / 2$ & $99.8(78.9)$ & $99.2(95.2)$ & $99.7(84.6)$ \\
\hline \multicolumn{4}{|l|}{ Refinement } \\
\hline Resolution $(\AA)$ & $50-2.50$ & $50-2.60$ & $50-2.20$ \\
\hline No. of unique reflections & 72,227 & 78,263 & 110,839 \\
\hline \multirow[t]{2}{*}{$R_{\text {work }} / R_{\text {free }}{ }^{b}$} & $0.153(0.236)$ & $0.156(0.182)$ & $0.155(0.239)$ \\
\hline & 0.209 (0.309) & $0.216(0.281)$ & $0.196(0.313)$ \\
\hline \multicolumn{4}{|l|}{ No. of atoms } \\
\hline Protein & 15,218 & 14,150 & 15,166 \\
\hline Ligand & 78 & N/A & 78 \\
\hline Solvent & 43 & 80 & 81 \\
\hline Water & 402 & 685 & 1,032 \\
\hline \multicolumn{4}{|l|}{ B factors $\left(\AA^{2}\right)$ (overall) } \\
\hline Protein & 52.02 & 35.91 & 45.54 \\
\hline Ligand & 43.02 & N/A & 37.57 \\
\hline Solvent & 75.68 & 69.15 & 53.89 \\
\hline Water & 43.25 & 34.40 & 43.55 \\
\hline \multicolumn{4}{|l|}{ RMSD } \\
\hline Bond lengths $(\AA)$ & 0.007 & 0.007 & 0.007 \\
\hline Bond angles $\left({ }^{\circ}\right)$ & 0.91 & 1.19 & 1.17 \\
\hline Ramachandran favored (\%) & 97.90 & 96.00 & 96.85 \\
\hline Ramachandran allowed (\%) & 2.10 & 3.78 & 3.04 \\
\hline Ramachandran outliers (\%) & 0 & 0.22 & 0.10 \\
\hline Rotamer outliers (\%) & 0.79 & 1.80 & 0.83 \\
\hline \multicolumn{4}{|c|}{$\begin{array}{l}\text { RMSD, root-mean-square deviation. } \\
\text { aHighest-resolution shell shown in parentheses. }\end{array}$} \\
\hline${ }^{\mathrm{b}} \mathrm{R} f \mathrm{ree}$ was calculated with 1 & data or no greater than 2 & foctione whichover is & \\
\hline
\end{tabular}




\begin{tabular}{|c|c|c|c|}
\hline Data name & $\begin{array}{l}\text { Cryptococcus neoformans } \\
\text { Ethyl-AMP } \\
\text { PDB 7KNO }\end{array}$ & $\begin{array}{l}\text { Cryptococcus neoformans } \\
\text { Butyl-AMP } \\
\text { PDB 7KNP }\end{array}$ & $\begin{array}{l}\text { Coccidioides } \\
\text { immitis } \\
\text { Propyl-AMP } \\
\text { PDB 7KQ6 }\end{array}$ \\
\hline \multicolumn{4}{|l|}{ Data collection } \\
\hline Wavelength $(\AA)$ & 0.9787 & 0.9787 & 0.9787 \\
\hline Space group & P 1211 & P 1211 & P 1211 \\
\hline \multicolumn{4}{|l|}{ Cell dimensions } \\
\hline$a, b, c(\AA)$ & 72.69184 .784 .84 & 72.25184 .6685 .13 & 106.98116 .08107 .33 \\
\hline$\alpha, \beta, \gamma\left({ }^{\circ}\right)$ & 90.093 .990 .0 & 90.093 .890 .0 & 90.0119 .890 .0 \\
\hline Resolution $(\AA)$ & $50-1.80(1.85-1.80)$ & $50-2.25(2.31-2.25)$ & $50-1.80(1.85-1.80)$ \\
\hline No. of unique reflections & $205,272(15,138)$ & $103,490(7,597)$ & $201,793(15,201)$ \\
\hline $\mathrm{R}_{\text {merge }}(\%)$ & $5.2(51.5)$ & $9.0(54.7)$ & $4.8(54.5)$ \\
\hline $\mathrm{I} / \sigma(\mathrm{I})$ & $15.12(2.31)$ & $10.14(2.60)$ & $15.73(2.10)$ \\
\hline Completeness (\%) & $99.8(99.9)$ & $98.4(97.9)$ & $95.8(97.9)$ \\
\hline Redundancy & $3.8(3.7)$ & $3.9(3.9)$ & $3.1(3.0)$ \\
\hline $\mathrm{CC}^{1} / 2$ & $99.9(84.9)$ & $99.5(81.0)$ & $99.9(74.4)$ \\
\hline \multicolumn{4}{|l|}{ Refinement } \\
\hline Resolution $(\AA)$ & $50-1.80$ & $50-2.25$ & $50-1.80$ \\
\hline No. of unique reflections & 205,190 & 103,450 & 201,774 \\
\hline$R_{\text {work }} / R_{\text {free }}$ & $0.164(0.261)$ & $0.176(0.235)$ & $0.162(0.240)$ \\
\hline & $0.190(0.285)$ & $0.213(0.267)$ & $0.185(0.288)$ \\
\hline \multicolumn{4}{|l|}{ No. of atoms } \\
\hline Protein & 14,987 & 13,988 & 14,057 \\
\hline Ligand & 75 & 81 & 78 \\
\hline Solvent & 57 & 58 & 128 \\
\hline Water & 1,761 & 822 & 1,556 \\
\hline \multicolumn{4}{|l|}{ B factors $\left(\AA^{2}\right)$ (overall) } \\
\hline Protein & 37.82 & 53.84 & 31.65 \\
\hline Ligand & 28.84 & 51.53 & 24.79 \\
\hline Solvent & 46.77 & 52.46 & 34.87 \\
\hline Water & 39.26 & 44.22 & 36.39 \\
\hline \multicolumn{4}{|l|}{ RMSD } \\
\hline Bond lengths $(\AA)$ & 0.008 & 0.007 & 0.007 \\
\hline Bond angles $\left({ }^{\circ}\right)$ & 0.90 & 0.83 & 0.80 \\
\hline Ramachandran favored (\%) & 96.86 & 96.18 & 95.88 \\
\hline Ramachandran allowed (\%) & 2.98 & 3.71 & 4.12 \\
\hline Ramachandran outliers (\%) & 0.16 & 0.11 & 0 \\
\hline Rotamer outliers (\%) & 0.13 & 0.85 & 0.55 \\
\hline
\end{tabular}

RMSD, root-mean-square deviation.

aHighest-resolution shell shown in parentheses.

${ }^{\mathrm{b}} \mathrm{Rfree}$ was calculated with $10 \%$ of the data or no greater than 2,000 reflections, whichever is lesser. 


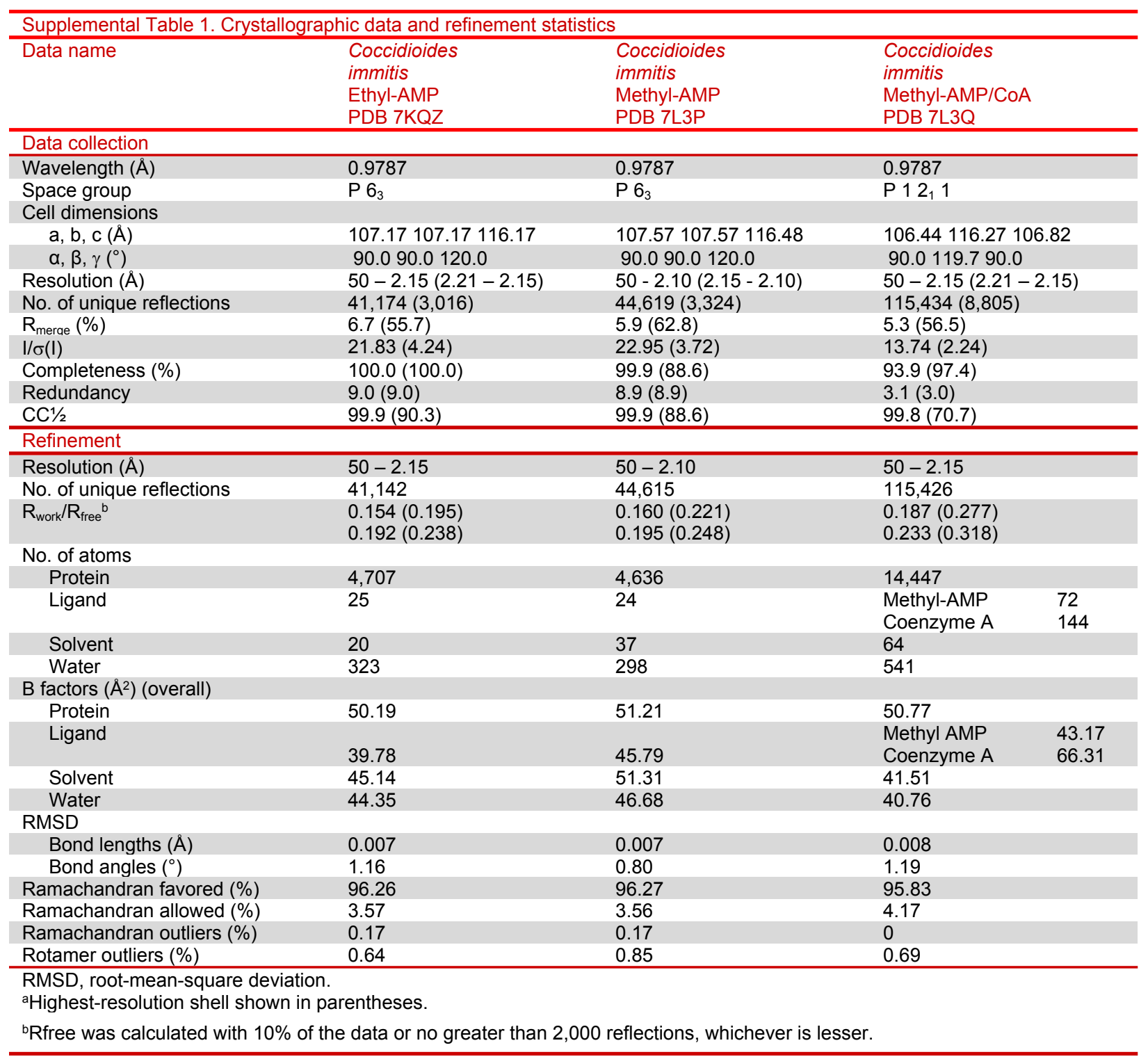




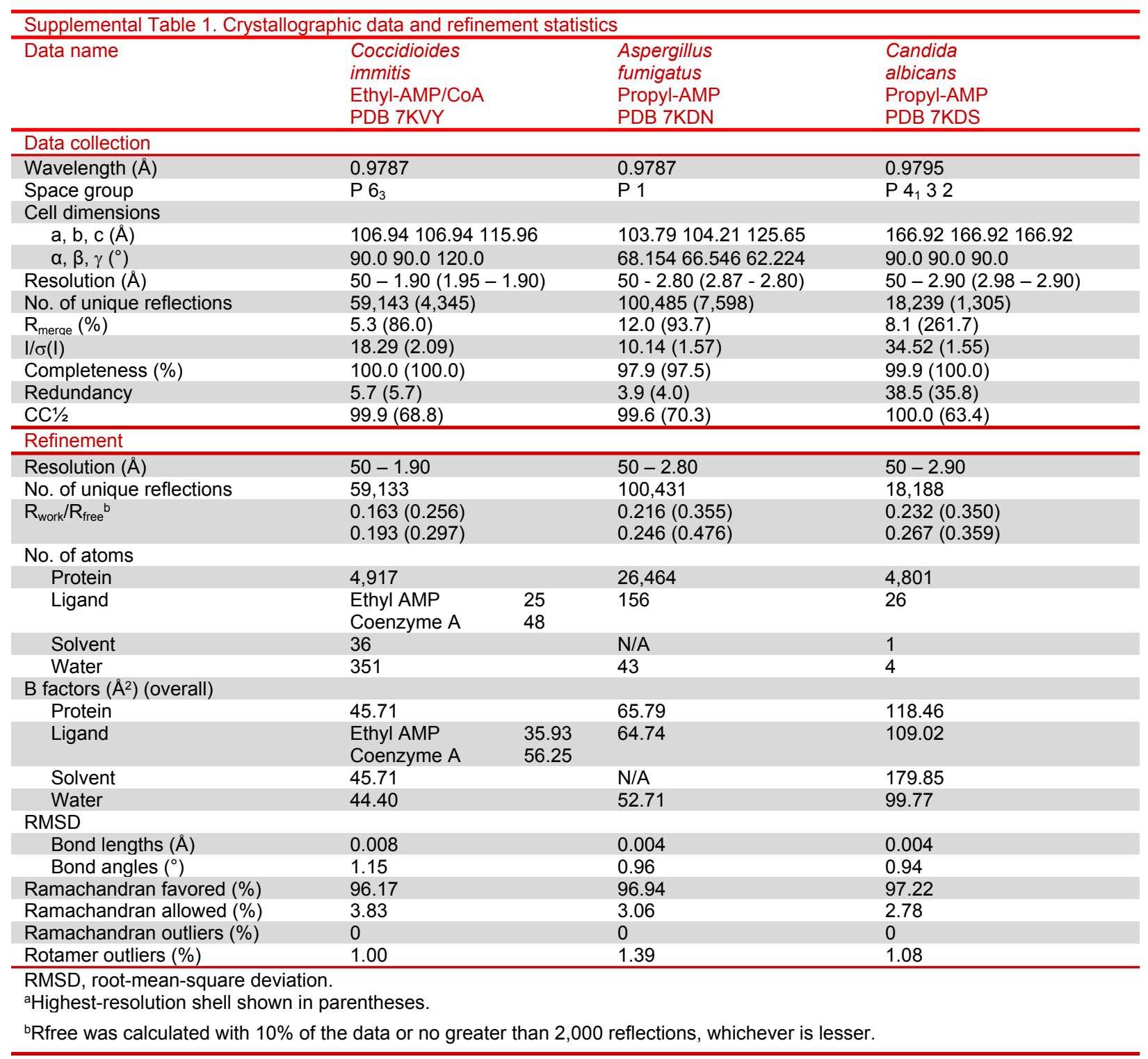




\begin{tabular}{|c|c|}
\hline Data name & $\begin{array}{l}\text { Coccidioides } \\
\text { posadasii } \\
\text { Propyl-AMP } \\
\text { PDB 7KCP }\end{array}$ \\
\hline \multicolumn{2}{|l|}{ Data collection } \\
\hline Wavelength $(\AA)$ & 0.9787 \\
\hline Space group & $\mathrm{P} 6_{3}$ \\
\hline \multicolumn{2}{|l|}{ Cell dimensions } \\
\hline$a, b, c(\AA)$ & 107.17107 .17116 .17 \\
\hline$\alpha, \beta, \gamma\left({ }^{\circ}\right)$ & 90.090 .0120 .0 \\
\hline Resolution $(\AA)$ & $50-2.15(2.21-2.15)$ \\
\hline No. of unique reflections & $41,158(3,025)$ \\
\hline$R_{\text {merge }}(\%)$ & $8.1(58.1)$ \\
\hline $\mathrm{I} / \sigma(\mathrm{I})$ & $20.04(4.20)$ \\
\hline Completeness (\%) & $100.0(100.0)$ \\
\hline Redundancy & $9.0(9.0)$ \\
\hline CC $1 / 2$ & $99.9(90.5)$ \\
\hline \multicolumn{2}{|l|}{ Refinement } \\
\hline Resolution $(\AA)$ & $50-2.15$ \\
\hline No. of unique reflections & 41,145 \\
\hline$R_{\text {work }} / R_{\text {free }}{ }^{b}$ & $\begin{array}{l}0.154(0.195) \\
0.192(0.238)\end{array}$ \\
\hline \multicolumn{2}{|l|}{ No. of atoms } \\
\hline Protein & 4,604 \\
\hline Ligand & 26 \\
\hline Solvent & 45 \\
\hline Water & 371 \\
\hline \multicolumn{2}{|l|}{ B factors $\left(\AA^{2}\right)$ (overall) } \\
\hline Protein & 42.88 \\
\hline Ligand & 34.32 \\
\hline Solvent & 44.80 \\
\hline Water & 41.07 \\
\hline \multicolumn{2}{|l|}{ RMSD } \\
\hline Bond lengths $(\AA)$ & 0.007 \\
\hline Bond angles $\left({ }^{\circ}\right)$ & 1.16 \\
\hline Ramachandran favored (\%) & 96.26 \\
\hline Ramachandran allowed (\%) & 3.57 \\
\hline Ramachandran outliers (\%) & 0.17 \\
\hline Rotamer outliers (\%) & 0.64 \\
\hline \multicolumn{2}{|c|}{$\begin{array}{l}\text { RMSD, root-mean-square deviation. } \\
\text { aHighest-resolution shell shown in parenthe }\end{array}$} \\
\hline${ }^{\mathrm{b}} \mathrm{R} f \mathrm{ree}$ was calculated with 1 & data or no greater than 2,000 reflections, whichever is lesser. \\
\hline
\end{tabular}

\section{Supplementary Table 1.}




\begin{tabular}{cccccccc}
\hline & \multicolumn{7}{c}{ Species } \\
\cline { 2 - 8 } Species & $C n$ & $A f$ & $C a$ & $C i$ & $C p$ & Sc & Se \\
\hline C. neoformans $(C n)$ & - & $63.13 \%$ & $58.95 \%$ & $59.97 \%$ & $59.65 \%$ & $56.50 \%$ & $51.03 \%$ \\
A. fumigatus $(A f)$ & $0.61 \AA$ & - & $63.29 \%$ & $82.26 \%$ & $82.71 \%$ & $62.99 \%$ & $51.10 \%$ \\
C. albicans $(C a)$ & $0.56 \AA$ & $0.53 \AA$ & - & $61.24 \%$ & $61.24 \%$ & $59.97 \%$ & $49.84 \%$ \\
C. immitis $(C i)$ & $0.55 \AA$ & $0.30 \AA$ & $0.51 \AA$ & - & $99.13 \%$ & $63.41 \%$ & $50.94 \%$ \\
C. posadasii $(C p)$ & $0.55 \AA$ & $0.30 \AA$ & $0.51 \AA$ & $0.11 \AA$ & - & $63.26 \%$ & $51.27 \%$ \\
S. cerevisiae $(\mathrm{Sc})$ & $0.60 \AA$ & $0.55 \AA$ & $0.56 \AA$ & $0.56 \AA$ & $0.55 \AA$ & - & $46.55 \%$ \\
S. enterica $(\mathrm{Se})$ & $0.66 \AA$ & $0.71 \AA$ & $0.76 \AA$ & $0.66 \AA$ & $0.61 \AA$ & $0.72 \AA$ & - \\
\hline
\end{tabular}

Supplementary Table 2. Structural and Sequence comparison of Acs1. Sequence alignment performed by $\mathrm{NCBI}$ blast pairwise alignment (Ref) with percent identity shown in the upper right section of the table. Alignment performed by PyMol (Ref) of Ca atoms in the N-terminal extension and N-terminal domain of pairwise species expressed as RMSD $(\AA)$ in the bottom left section of the table. 
Crne J9VFT1

Coim J3KJM0

Copo C5PGB4

Asfu Q4WQ02

Caal Q8NJN3

Sace Q01574
Crne J9VFT1 Coim J3KJM0 Copo C5PGB4 Asfu Q4WQ02 Caal Q8NJN3 Sace Q01574

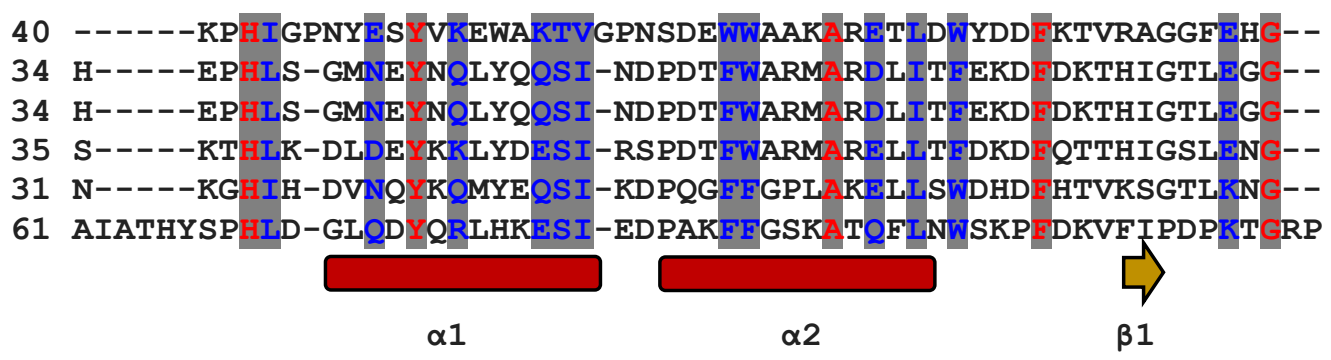

A1

Crne J9VFT1 Coim J3KJM0 Copo C5PGB4 Asfu Q4WQ02 Caal Q8NJN3 Sace Q01574
$1 \mathrm{M}$

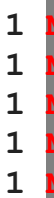

- GKTEV-APGVHHVHPLPDSVPESEDLFAP
-ETPAEP-KLPVVVEAHQVDTFDVPGVFYEN

-ETPAEP-KPPVVVEAHQVDTFDVPGVFYEN

DGDVKPAKPAVVLEANDVDTFHVPKAFYEK

1 MSPSAVQSSKLEEQSSEIDKLKAKMSQSAATAQQKKEHEYEHLTSVKIVPQRPISDRLQP

PPRMQGKEGRP

\section{HP HP} . $\alpha 1$

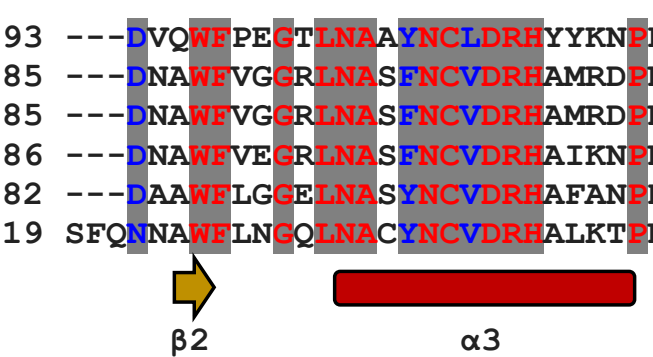

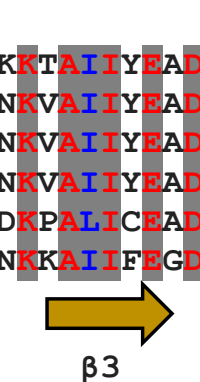

$\beta 3$

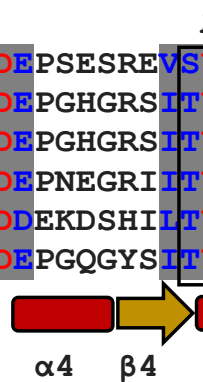

$\alpha 4 \quad \beta 4$ . .

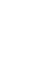

A2

1

2

Crne J9VFT1 150 Coim J3KJM0 142 Copo C5PGB4 142 Asfu Q4WQ02 143 Caal Q8NJN3 139 Sace Q01574 179

$\alpha 5$

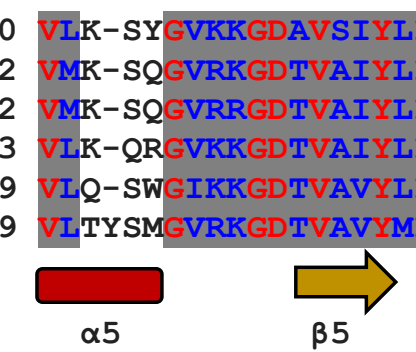

$\beta 5$

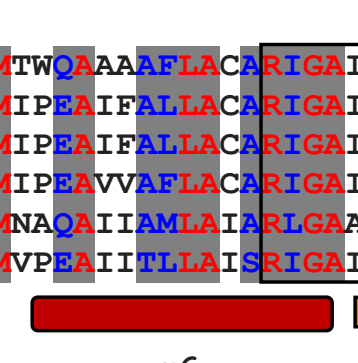

$\alpha 6$ $\circ 0$

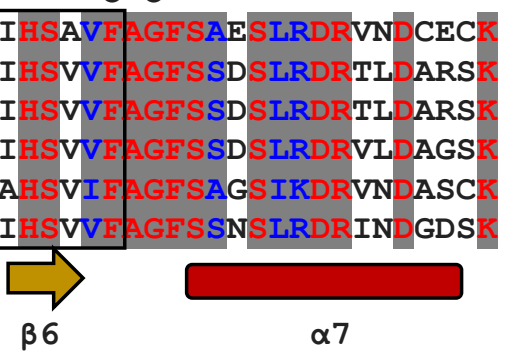

1

2

$\circ$

Crne J9VFT1 209 VLITTDEGRRGGKTIATKQIVDAAIQQCPLVENVLVLRRT-GNKVPMTEGRDKWWDEECA Coim J3KJMO 201 FIITTDEGKRGGKVIGTKKIVDEALKOCPDVTNCLVFKRT-GADVPWTKGRDLWWHEEVD Copo C5PGB4 201 FIITTEGKRGGKVIGTKKIVDEAIKOCPDVTSCLVFKRT-GADVPWTKGRDLWWHEEVE Asfu Q4WQ02 202 VVITTDEGKRGGKVIGTKRIVDEAIKQCPDVTSVLVYKRT-GAEVPWTNGRDIWWHEEVE Caal Q8NJN3 198 ALITCDEGKRGGRTNIKKLCDEALVDCPTVEKVLVYKRTNNPEIHLTEGRDYYWDVETA Sace Q01574 239 VVITTDESNRGGKVIETKRIVDDALRETPGVRHVLVYRKTNNPSVAFHAPRDLDWATEKK

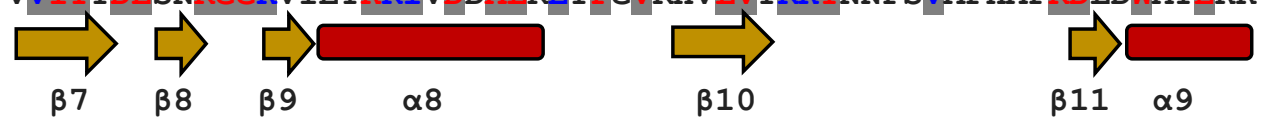


2

Crne J9VFT1 268 KMPAYCPCERMASEDPLFILYTSGTGKPKGVVSTAGYLLGTALTLKYVFDAHPDDRFA Coim J3KJM0 260 KYPNYLPAESMD SEDPLFLLYTSGSTGKPKGVMHTTAGYLVGAAATGKYVFDIHPADRFF Copo C5PGB4 260 KYPNYLPAESMD SEDPLFLLYTSGTGKPKGVMHTTAGYLVGAAATGKYVFDIHPADRFF Asfu Q4WQ02 261 KYPCYVAPESMSSEDPLFLITSGSTGKPKGMMTTAGYLLGAAMTGKYVFDI HDDDRFF Caal Q8NJN3 258 KFPGYLPPVSVNSEDPLFLLYTSGSTGTPKGVVHSTAGYLLGAALSTKYIFDIHPEDILF Sace Q01574 299

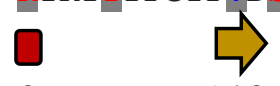

$\alpha 9$ $\beta 12$

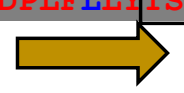

$\beta 13$

$\mathrm{A} 4$

1

$\varnothing \times x$

200 ○‡»

Crne J9VFT1 328 CMADI IWITG ISYIIYGPIANGITTAVFESTPVYPTPSRYWDFVDKWKATQLYTAPTAIR Coim J3KJMO 320 CGGDVGWITG ITYVVYAPILLGCTIVVFESTPAYPNFSRYWDVIEKHKVIQFYVAPTALR COPO C5PGB4 320 CGGDVGWITG ITYVVYAPILLGCT IVVFESTPAYPNFSRYWDVIEKHKVTQFYVAPTALR Asfu Q4WQ02 321 CGGDVGWITG TTYVYAPILLGCS VVVFESTPAYPNFSRYWDVIEKHKVTQFYVAPTALR Caal Q8NJN3 318 TAGDVGWITG FTYALYGPILLGVPII IFEGTPAYPDYGRFWOIVEKHKATHFYVAPTALR Sace 201574359 TAGDIGWITG TYVVYGPLLYGCAILVFEGTPAYPNYSRYWDIIDEHKVTQFYVAPTALR
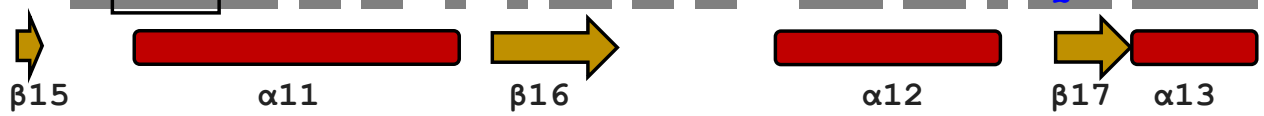

1

2
Crne J9VFT1 388 LIRRMGEDHVKNHDLSS RVLGSVGEPINPEANHWYNDFAGKNQCATVDTYWMTEGSIS Coim J3KJMO 380 LIKRA GDHI-NHEMKDLRILGSVGEPIAAEVWKWYHEVVGKROAHIVDT YWQ TE TISHV Copo C5PGB 380 LLKRA GDHHI-NHEMKDLRI LGSVGEPIAAEVWKWYHEVVGKRQAHIVDTYWQTETGSHV Asfu Q4WQ02 381 LLKRA GDEHI-HHKMEHLRI LGSVEPIAAEVWKWYFKVGKEEAHICDTYWQTEGSH Caal Q8NJN3 378 LLRKA GEQEIAKYDLSSLRTLGSVGEPISPDI WEWYNEFVGKNQCHISDT YWQTESGSHL Sace Q01574 419

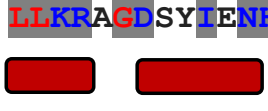

$\alpha 13$

$\alpha 14$
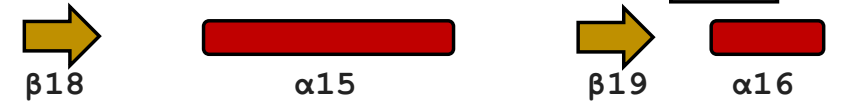

A6

Crne J9VFT1 448 IAPL-PGAISTKPGSATFPFFGMDVDIIDPQTGQVLEGNDVEGVIVARRPWPSIARTVYR Coim J3KJM0 439 ITPI-GGITPTKPGSASLPFFGIDPVILDPVTGAEIPGNDVEGI AFRKPWPSMARTVWG Copo C5PGB4 439 ITPL-GEITPTKPGSASLPFFGIDPVILDPVTGAEIPGNDVEGI AFRKPWPSMARTVWG Asfu Q4WQ02 440 ISPL-GGITSTKPGSASLPFFGIEPAIIDPVSGEEISGNDVEGV AFKQPWPSMARTVWG Caal Q8NJN3 438 IAPI-AGVVPNKPGSASYPFFGIDAALIDPVTGVEIEGNDAEGV AIKDHWPSMARTVYK Sace Q01574 479 VTPIAGGVTPMKPGSASFPFFGIDAVVLDPNTGEELNTSHAEGV AVKAANPSFARTIWK

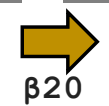

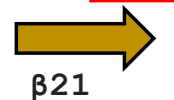

$\beta 21$

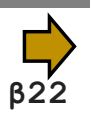




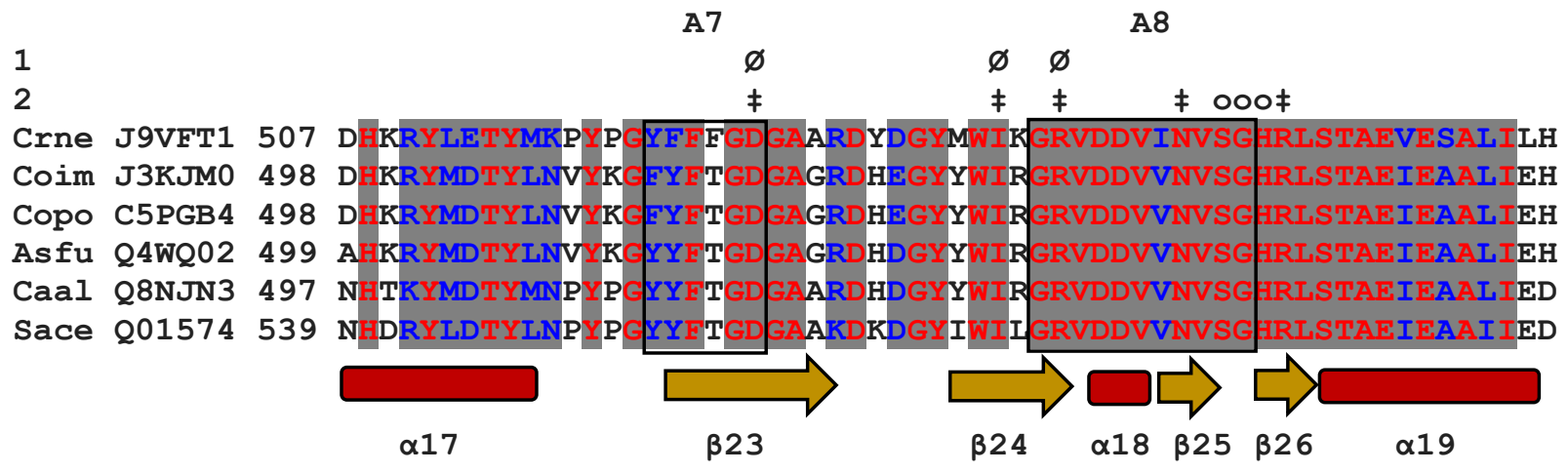

A9

1

2 Crne J9VFT1 567 KGVAFTAVVGCADDLTGQAVYAFVTMKPEFDLK---ATKEADLSKETAIQVRKVEGPFAA Coim J3KJMO 558 HCVAEAAVVGVP PLTGQAVHAFVALKSGN-------DNREQLQKELIMQVRKS IGPFAA Copo C5PGB4 558 HCVAFAAVVGVPDPLTGQAVHAFVALKSGN------DNREQLR ELIMQVRKSEGPFAA Asfu Q4WQ02 559 HQVAEAAVVGIADELTGQAVNAFVSLKDGN-------ETTEQVRKDLVMVRKS IGPFAA Caal Q8NJN3 557 KKVSEAAVVGIHDDITGQAVIAYVALKEGNS-----DEDSEGLRKELVLQVRKT EGPAA Sace 201574599 PIVAECAVVGFNDDLTGQVAAFVVLKNKSSWSTATDDELQDIKKHLVETVRKDEGPFA

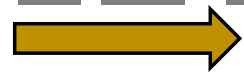

$\beta 27$

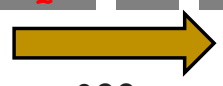

$\beta 28$

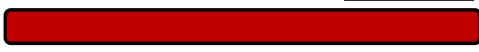

$\alpha 20$

A10

1

2

Crne J9VFT1 624

Coim J3KJM0 611

Copo C5PGB4 611

Asfu Q4WQ02 612

Caal Q8NJN3 612

Sace 201574659

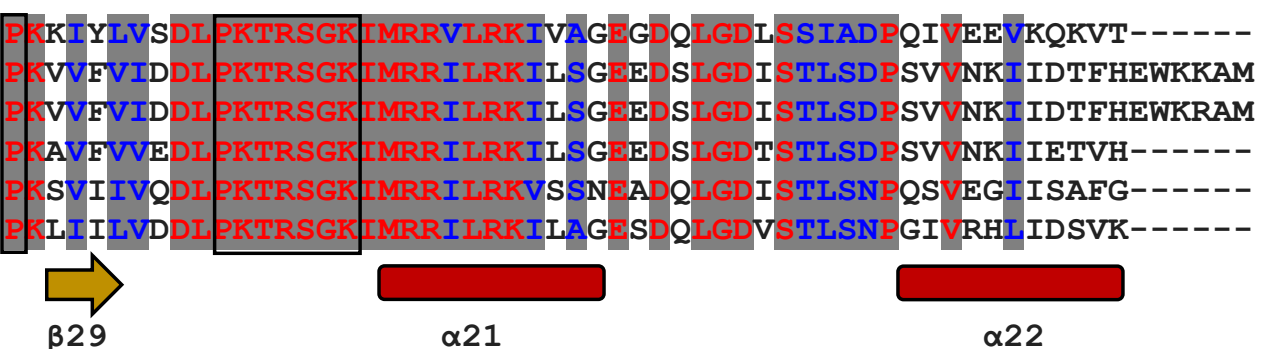

Crne J9VFT1 $678------G S----------A 680$

Coim J3KJM0 671 AAAAAAAAAVSATAPPNSTTG 691

Copo C5PGB4 671 AAAAAAAAAVSATAPPNSTTG 691

Asfu Q4WQ02 666 ----AARA------------K 670

Caal Q8NJN3 $666----A Q F G K----------K 671$

Sace Q01574 713 -----------------L 713

Supplemental Table 3. Alignment of fungal Acs1. Multiple sequence alignment by MAFFT (Ref) with Cryptococcus neoformans (Uniprot ID J9VFT1), Aspergillus fumigatus (Uniprot ID Q4WQ02), Candida albicans (Uniprot ID Q8NJN3), Coccidioides immitis (Uniprot ID J3KJMO), Coccidioides posadasii (Uniprot ID C5PGB4), and Saccharomyces cerevisiae (Uniprot ID Q01574). Secondary structure shown below the alignment as red rectangles (alpha helices) and 
yellow arrows (beta strands). Identical residues are in red font with gray background and chemically similar compounds are in blue font with gray background. Boxes over the alignment indicate conserved Acs1 enzyme family motifs labeled A1-A10. Symbols above the alignment indicate ligand or substrate interacting residues with "1" for the Acetylation reaction conformation $(\bullet)$ ATP only, (×) Acetyl-AMP only and, ( $\varnothing)$ both ligands; "2" for the thioesterification reaction conformation. (o) Coenzyme A only, (†) Acetyl-AMP only, (») both. 


\begin{tabular}{ccc}
\hline & \multicolumn{2}{c}{ Compound $\mathbf{I C}_{50}(\boldsymbol{\mu M})$} \\
\cline { 2 - 3 } Species & ethyl-AMP & butyl-AMP \\
\hline C. neoformans & 2.10 & $>50$ \\
A. fumigatus & 0.06 & $>50$ \\
C. albicans & 0.29 & $>50$ \\
C. immitis & 0.06 & $>50$ \\
C. posadasii & 0.07 & $>50$ \\
\hline
\end{tabular}

Supplemental Table 4. Potency of ethyl-AMP and butyl-AMP across fungal Acs1 recombinant proteins 


\section{Synthetic Procedures for the Preparation of AMP alkyl esters, AMS and AcAMS.}

All chemicals were purchased from Sigma Aldrich and were used without any further purification. 5'-O-Sulfamoyl-2', 3'-O-isopropylideneadenosine, N-acetoxysuccinimide, AMS and Ac-AMS were prepared by the method of Qiao (1). All tested compounds have purity of $>95 \%$ as determined by HPLC analysis (UV detection @254 nM). Purification of compounds was done on a Biotage ${ }^{\circledR}$ Isolera using a Biotage $®$ SNAP cartridge KP-Sil $50 \mathrm{~g}$. Purity of compounds was determined using an Agilent HPLC utilizing a C-18 column (Waters Nova-Pak; $3.9 \times 100 \mathrm{~mm}$ ) with the following method: Solvent $A=\mathrm{H}_{2} \mathrm{O}(0.1 \%$ TFA), Solvent $B=\mathrm{MeCN} ; 0$ to $20 \mathrm{~min}$, $(10$ to $90 \% \mathrm{~B}), 20$ to 25 $\min \left(90\right.$ to $10 \% \mathrm{~B}$ ); detection was set at two wavelengths (254 and $280 \mathrm{~nm}$ ). ${ }^{1} \mathrm{H}$ and ${ }^{13} \mathrm{C}$ NMR were recorded on a BrukerAvance III 500 outfitted with a $5 \mathrm{~mm}$ BBFO Z-gradient probe. Chemical shifts $\delta$ are in ppm, and spectra are referenced using the residual solvent peak. The following abbreviations are used: singlet (s), doublet (d), triplet (t), quartet (q), double doublet (dd), quintet (quin), multiplet (m), broad signal (br s). High-resolution mass spectra (HRMS) were obtained on a Bruker Maxis Plus Quadrupole Time-of-Flight (QTOF).

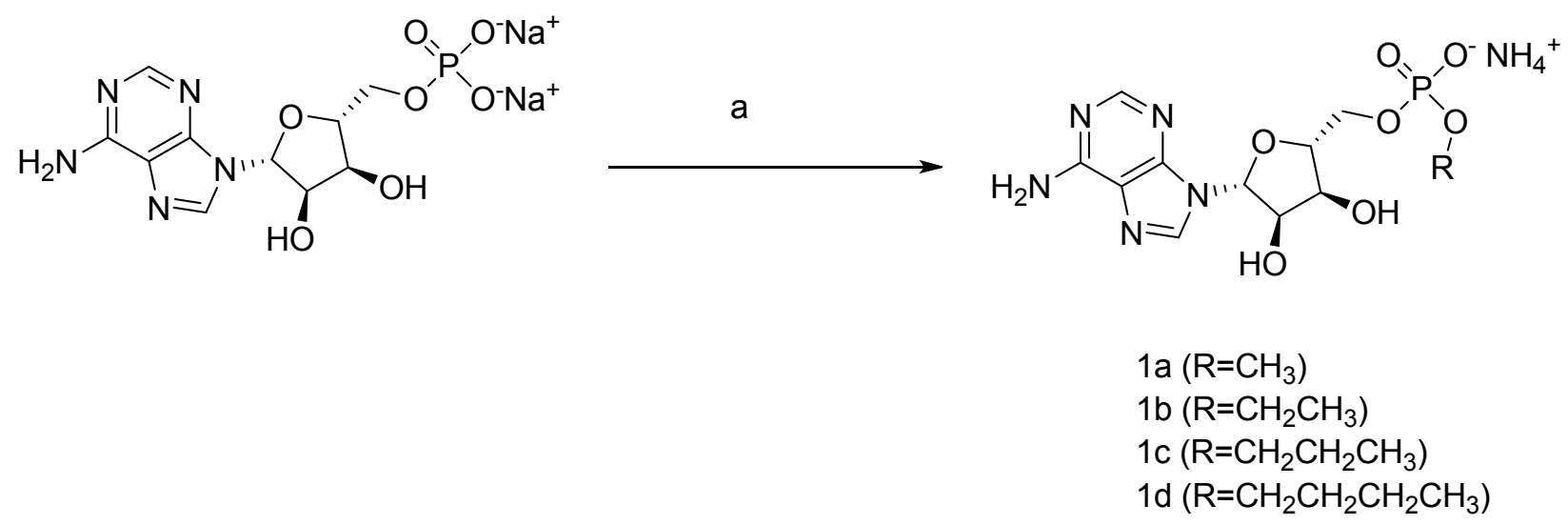

Reagent and reaction conditions: (a) Corresponding alcohol, EDC (5.0 eq), rt, $24 \mathrm{~h}$.

\section{General procedure for preparation of adenosine 5'-alkyl phosphate (1a-1d)}

To a stirred solution of adenosine monophosphate disodium salt $(1.00 \mathrm{~g}, 2.56 \mathrm{mmol})$ in $150 \mathrm{~mL}$ of corresponding alcohol was added EDC (2.45 g, $12.78 \mathrm{mmol})$. The reaction was stirred for 24 
hr. followed by removal of the solvent under reduced under pressure. The resulting solid was purified by column chromatography on a Biotage Isolera using a Biotage SNAP cartridge KP-Sil $50 \mathrm{~g}$ using a linear gradient of $80: 20$ to $70: 30\left(\mathrm{CH}_{2} \mathrm{Cl}_{2} / \mathrm{MeOH}\right.$ with $\left.4 \% \mathrm{NH}_{4} \mathrm{OH}\right)$ to afford the corresponding compounds $\mathbf{1 a - 1 d}$ as white solids.

\section{Adenosine 5'-methyl phosphate (Methyl-AMP) (1a)}

Yield; 63\%, ${ }^{1} \mathrm{H}$ NMR (500 MHz, DMSO-d6) $\delta 8.46(\mathrm{~s}, 1 \mathrm{H}), 8.15(\mathrm{~s}, 1 \mathrm{H}), 7.31(\mathrm{~s}, 2 \mathrm{H}), 5.92(\mathrm{~d}, \mathrm{~J}=$ $6.0 \mathrm{~Hz}, 1 \mathrm{H}), 5.54(\mathrm{~s}, 1 \mathrm{H}), 5.43(\mathrm{~s}, 1 \mathrm{H}), 4.60(\mathrm{t}, \mathrm{J}=6.9,1 \mathrm{H}), 4.20(\mathrm{t}, J=4.0 \mathrm{~Hz}), 4.02(\mathrm{q}, \mathrm{J}=4.0 \mathrm{~Hz})$, $3.84(\mathrm{~m}, 1 \mathrm{H}), 3.80(\mathrm{~m}, 1 \mathrm{H}), 3.31(\mathrm{~d}, J=10.5 \mathrm{~Hz}, 3 \mathrm{H}),{ }^{13} \mathrm{C}$ NMR (125 MHz, DMSO-d6), 156.4, 153.1, 150.1, 119.3, 87.4, 84.4, 74.4, 71.3, 65.0, 52.1, $\mathrm{HRMS}$ for $\mathrm{C}_{11} \mathrm{H}_{16} \mathrm{~N}_{5} \mathrm{O}_{7} \mathrm{P}[\mathrm{M}+\mathrm{H}]^{+}$calculated; 362.0866, found; 362.0855

\section{Adenosine 5'-ethyl phosphate (Ethyl-AMP) (1b)}

Yield; 46\%, ${ }^{1} \mathrm{H}$ NMR (500 MHz, DMSO-d6) $\delta 8.46$ (s, 1H), 8.15 (s, 1H), 7.30 (s, 2H), 5.92 (d, J= $6.0 \mathrm{~Hz}, 1 \mathrm{H}), 5.56(\mathrm{~s}, 1 \mathrm{H}), 5.55(\mathrm{~s}, 1 \mathrm{H}), 4.59(\mathrm{t}, \mathrm{J}=6.9,1 \mathrm{H}), 4.19(\mathrm{t}, J=4.0 \mathrm{~Hz}), 4.03(\mathrm{q}, \mathrm{J}=4.0 \mathrm{~Hz})$, $3.87(\mathrm{~m}, 1 \mathrm{H}), 3.79(\mathrm{~m}, 1 \mathrm{H}), 3.68(\mathrm{~m}, 2 \mathrm{H}), 1.08(\mathrm{t}, \mathrm{J}=7.0 \mathrm{~Hz}, 3 \mathrm{H}),{ }^{13} \mathrm{C}$ NMR $(125 \mathrm{MHz}, \mathrm{DMSO}-d 6)$, $156.4,153.1,150.1,139.8,119.3,87.4,84.3,74.3,71.2,64.9,60.3,17.0, \mathrm{HRMS}$ for $\mathrm{C}_{12} \mathrm{H}_{18} \mathrm{~N}_{5} \mathrm{O}_{7} \mathrm{P}$ $[\mathrm{M}+\mathrm{H}]^{+}$calculated; 376.1023, found; 376.1033

\section{Adenosine 5'- propyl phosphate (Propyl-AMP) (1c)}

Yield; 43\%, ${ }^{1} \mathrm{H}$ NMR (500 MHz, DMSO-d6) $\delta 8.44$ (s, 1H), 8.15 (s, 1H), 7.30 (s, 2H), 5.91 (d, J= $6.0 \mathrm{~Hz}, 1 \mathrm{H}), 5.57(\mathrm{~s}, 1 \mathrm{H}), 5.56(\mathrm{~s}, 1 \mathrm{H}), 4.59(\mathrm{t}, \mathrm{J}=6.9,1 \mathrm{H}), 4.19(\mathrm{t}, J=4.0 \mathrm{~Hz}), 4.02(\mathrm{q}, J=4.0 \mathrm{~Hz})$, $3.87(\mathrm{~m}, 1 \mathrm{H}), 3.78(\mathrm{~m}, 1 \mathrm{H}), 3.58(\mathrm{q}, \mathrm{J}=6.7 \mathrm{~Hz}, 2 \mathrm{H}), 1.45(\mathrm{~m}, 2 \mathrm{H}), 0.79(\mathrm{t}, \mathrm{J}=7.5 \mathrm{~Hz}, 3 \mathrm{H}),{ }^{13} \mathrm{C}$ NMR (125 MHz, DMSO-d6), 156.4, 153.1, 150.0, 139.8, 119.3, 87.5, 84.2, 74.2, 71.2, 66.4, 65.0, 24.0, 10.8, HRMS for $\mathrm{C}_{13} \mathrm{H}_{20} \mathrm{~N}_{5} \mathrm{O}_{7} \mathrm{P}[\mathrm{M}+\mathrm{H}]^{+}$calculated; 390.1179 , found; 390.1192 


\section{Adenosine 5'- butyl phosphate (Butyl-AMP) (1d)}

Yield; 35\%, ${ }^{1 H}$ NMR (500 MHz, DMSO-d6) $\delta 8.44$ (s, 1H), 8.15 (s, 1H), 7.29 (s, 2H), 5.91 (d, J= $6.0 \mathrm{~Hz}, 1 \mathrm{H}), 5.52(\mathrm{~s}, 1 \mathrm{H}), 5.42(\mathrm{~s}, 1 \mathrm{H}), 4.58(\mathrm{t}, \mathrm{J}=6.9,1 \mathrm{H}), 4.19(\mathrm{t}, J=4.0 \mathrm{~Hz}), 4.02(\mathrm{q}, J=4.0$ $\mathrm{Hz}), 3.86(\mathrm{~m}, 1 \mathrm{H}), 3.78(\mathrm{~m}, 1 \mathrm{H}), 3.61(\mathrm{q}, \mathrm{J}=6.7 \mathrm{~Hz}, 2 \mathrm{H}), 1.43(\mathrm{~m}, 2 \mathrm{H}), 1.24(\mathrm{~m}, 2 \mathrm{H}), 0.81(\mathrm{t}, \mathrm{J}=$ $7.5 \mathrm{~Hz}, 3 \mathrm{H}),{ }^{13} \mathrm{C}$ NMR (125 MHz, DMSO-d6), 156.4, 153.1, 150.0, 139.9, 119.3, 87.5, 84.1, 74.2, 71.2, 65.1, 64.5, 32.9, 19.0, 14.1, HRMS for $\mathrm{C}_{14} \mathrm{H}_{22} \mathrm{~N}_{5} \mathrm{O}_{7} \mathrm{P}[\mathrm{M}+\mathrm{H}]^{+}$calculated; 404.1336, found; 404.1342
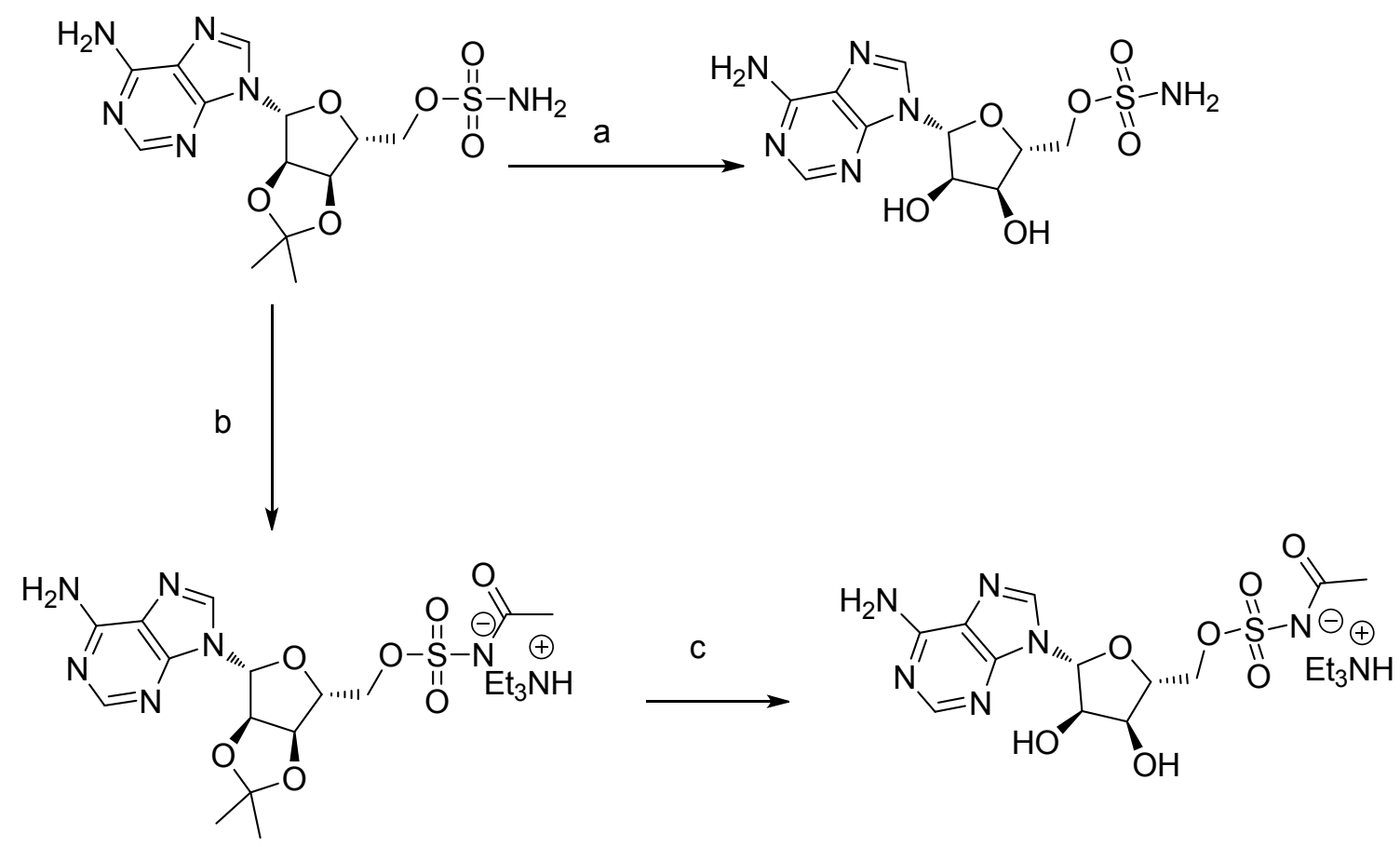

Reagent and reaction conditions: (a) TFA (80\%) (b) N-acetoxysuccinimide, $\mathrm{Cs}_{2} \mathrm{CO}_{3}$ (2eq), DMF (d) TFA (80\%).

\section{5'-O-Sulfamoyladenosine (AMS)}

To 5'-O-Sulfamoyl-2', 3'-O-isopropylideneadenosine (50 mg, $0.13 \mathrm{mmol}$ ) was added $80 \%$ aq TFA $(2 \mathrm{~mL})$. The resulting solution was stirred for $30 \mathrm{~min}$ at $0{ }^{\circ} \mathrm{C}$ then concentrated under reduced pressure. Purification by column chromatography on a Biotage Isolera using a gradient elution 15 to $20 \% \mathrm{MeOH}$ in $\mathrm{CH}_{2} \mathrm{Cl}_{2}$ afforded the title compound as a white solid. Yield; $40 \mathrm{mg}, 89 \%,{ }^{1} \mathrm{H} \mathrm{NMR}$ (500 MHz, DMSO-d6) ס, 8.30 (s, 1H), $8.16(\mathrm{~s}, 1 \mathrm{H}), 7.32$ (s, 2H), 7.60 (s, 2H), 5.94 (d, J= 5.30 Hz, 
1H), $5.44(d, J=5.20 \mathrm{~Hz}, 1 \mathrm{H}), 5.62(\mathrm{~d}, \mathrm{~J}=5.85 \mathrm{~Hz}, 1 \mathrm{H}), 4.63(\mathrm{~d}, \mathrm{~J}=5.25 \mathrm{~Hz}, 1 \mathrm{H}), 4.30(\mathrm{~d}, \mathrm{~J}=6.85$ $\mathrm{Hz}, 1 \mathrm{H}), 4.23-4.21(\mathrm{~m}, 2 \mathrm{H}), 4.17$ (d, J=1.55 Hz), ${ }^{13} \mathrm{C}$ NMR (75 MHz, DMSO-d6), 156.5, 153.1, $149.9,139.9,119.6,88.0,82.0,73.4,70.8,69.2$.

\section{5'-O-[N-acyl(sulfamoyl)]-2', 3'-O-isopropylideneadenosine triethylammonium salt.}

To a solution of $\mathrm{N}$-acetoxysuccinimide $(0.86 \mathrm{mmol})$ in DMF $(10 \mathrm{~mL})$ at $0{ }^{\circ} \mathrm{C}$ were added 5 - $\mathrm{O}$ sulfamoyl-2',3'-O-isopropylideneadenosine ${ }^{1}$ (1.29 mmol, 1.5equiv) and $\mathrm{Cs}_{2} \mathrm{CO}_{3}$ (1.72 mmol, 2.0 eq.). The reaction mixture was warmed to $\mathrm{rt}$ and stirred $16 \mathrm{~h}$. The reaction was concentrated under reduced pressure and the crude material was chromatographed on a Biotage Isolera using EtOAc/MeOH/Et ${ }_{3} \mathrm{~N}(70: 29: 1)$ to afford the title compound as a white solid. Yield; $87 \%$. ${ }^{1} \mathrm{H}$ NMR (300 MHz, DMSO-d6) $\delta 8.38(\mathrm{~s}, 1 \mathrm{H}), 8.15(\mathrm{~s}, 1 \mathrm{H}), 7.31(\mathrm{~s}, 2 \mathrm{H}), 6.15$ (d, J = 2.9 Hz, 1H), 5.35 (dd, $\mathrm{J}=2.9,6.1 \mathrm{~Hz}, 1 \mathrm{H}), 5.02(\mathrm{~d}, \mathrm{~J}=8.5 \mathrm{~Hz}, 1 \mathrm{H}), 4.37(\mathrm{~d}, \mathrm{~J}=2.5 \mathrm{~Hz}, 1 \mathrm{H}), 3.98(\mathrm{~d}, \mathrm{~J}=5.0 \mathrm{~Hz}, 2 \mathrm{H})$, $3.04(\mathrm{q}, \mathrm{J}=7.3 \mathrm{~Hz}, 6 \mathrm{H}), 1.72(\mathrm{~s}, 3 \mathrm{H}), 1.54(\mathrm{~s}, 3 \mathrm{H}), 1.32(\mathrm{~s}, 3 \mathrm{H}), 1.16(\mathrm{t}, \mathrm{J}=7.3 \mathrm{~Hz}, 9 \mathrm{H}),{ }^{13} \mathrm{C}$ NMR (75 MHz, $\left.\mathrm{CD}_{3} \mathrm{OD}\right), 179.2,157.3,155.9,149.0,140.1,120.0,113.9,90.4,85.2,84.3,80.4$, $68.2,43.2,26.1,25.1,23.7,7.9$.

\section{5'-O-[N-acyl(sulfamoyl)] adenosine triethylammonium salt (Ac-AMS)}

To 5'-O-[N-acyl(sulfamoyl)]-2', 3'-O-isopropylideneadenosine triethylammonium salt $(0.50 \mathrm{~g}$, $0.94 \mathrm{mmol}$ ) was added $80 \%$ aq TFA $(5 \mathrm{~mL})$. The resulting solution was stirred for 30 min at $0{ }^{\circ} \mathrm{C}$ then concentrated under reduced pressure. Purification by column chromatography on a Biotage Isolera using EtOAc/MeOH/Et ${ }_{3} \mathrm{~N}(70: 29: 1)$ afforded the title compound as a pale yellow solid. Yield; 0.42 g, 89\%. ${ }^{1} \mathrm{H}$ NMR (500 MHz, $\left.\mathrm{CD}_{3} \mathrm{OD}\right) \delta 8.52(\mathrm{~s}, 1 \mathrm{H}), 8.23(\mathrm{~s}, 1 \mathrm{H}), 6.11$ (d, J = $6.0 \mathrm{~Hz}$, 1H), $4.69(\mathrm{~s}, 1 \mathrm{H}), 4.42(\mathrm{~s}, 1 \mathrm{H}), 4.34-4.31(\mathrm{~m}, 3 \mathrm{H}), 3.20(\mathrm{q}, \mathrm{J}=7.0 \mathrm{~Hz}, 6 \mathrm{H}), 1.98(\mathrm{~s}, 3 \mathrm{H}), 1.31$ (t, J $=7.2 \mathrm{~Hz}, 9 \mathrm{H}),{ }^{1} \mathrm{H}$ NMR $(300 \mathrm{MHz}, \mathrm{DMSO}-\mathrm{d} 6) \delta 8.40(\mathrm{~s}, 1 \mathrm{H}), 8.14(\mathrm{~s}, 1 \mathrm{H}), 7.27(\mathrm{~s}, 2 \mathrm{H}), 5.91(\mathrm{~d}, \mathrm{~J}$ $=6.0 \mathrm{~Hz}, 1 \mathrm{H}), 5.46(\mathrm{~s}, 1 \mathrm{H}), 5.31(\mathrm{~s}, 1 \mathrm{H}), 4.60(\mathrm{~s}, 1 \mathrm{H}), 4.14(\mathrm{~s}, 1 \mathrm{H}), 4.07-3.99(\mathrm{~m}, 3 \mathrm{H}), 3.04(\mathrm{q}, \mathrm{J}$ 
$=7.0 \mathrm{~Hz}, 6 \mathrm{H}), 1.74(\mathrm{~s}, 3 \mathrm{H}), 1.14(\mathrm{t}, \mathrm{J}=7.2 \mathrm{~Hz}, 9 \mathrm{H}),{ }^{13} \mathrm{C}$ NMR (75 MHz, DMSO-d6), 175.5, 156.5, $153.1,150.1,139.8,119.3,93.6,87.2,83.2,74.1,71.4,67.6,46.2,26.9,9.5$.

\section{Crystallization conditions and procedures}

\section{Aspergillus fumigatus}

Aspergillus fumigatus ACS1 in complex with adenosine-5'-propylphosphate (propyl AMP) was crystallized at $10 \mathrm{mg} \mathrm{ml}-1$ in sparse matrix screen Morpheus (Molecular Dimensions) condition g9 (10\% w/v PEG 20000, 20\% v/v PEG MME 550, 0.02M carboxylic acids (sodium formate, ammonium acetate, trisodium citrate, sodium potassium L-tartrate, sodium oxamate, $0.1 \mathrm{M}$ bicine/Trizma base $\mathrm{pH} 8.5$ ), with $1 \mathrm{mM}$ propyl AMP and $1 \mathrm{mM}$ TCEP (2). No additional cryoprotectant was used.

\section{Candida albicans}

Candida albicans ACS2 was crystallized in complex with propyl AMP at $10 \mathrm{mg} / \mathrm{ml}$ in sparse matrix screen MCSG1 (Microlytic/Anatrace) condition g10 (100mM Mg-formate, 15\% (w/v) PEG 3350) with $1 \mathrm{mM}$ Propyl AMP and $1 \mathrm{mM}$ TCEP. $25 \%$ Ethylene glycol (v/v) was used as a cryoprotectant.

\section{Coccidioides posadasii}

Crystals of Coccidioides posadasii ACS1 in complex with propyl AMP were grown at $10 \mathrm{mg} / \mathrm{ml}$ ACS1 in sparse matrix screen Morpheus (Molecular Dimensions) condition a6 (10\% w/v PEG 8000, 20\% v/v ethylene glycol, 0.03M divalent cations ( $\mathrm{MgCl} 2, \mathrm{CaCl} 2), 0.1 \mathrm{M} \mathrm{MOPS/HEPES-Na}$ $\mathrm{pH} 7.5)$, with $1 \mathrm{mM}$ propyl-AMP and $1 \mathrm{mM}$ TCEP (2). No additional cryo-protectant was used.

\section{Coccidioides immitis}


Crystals of Coccidioides immitis ACS1 in complex with methyl AMP were grown at $10 \mathrm{mg} / \mathrm{ml}$ ACS1 in sparse matrix screen MCSG1 condition f11 $(0.1 \mathrm{M} \mathrm{HEPES/NaOH}, \mathrm{pH} 7.5,0.2 \mathrm{M}$ Ammonium sulfate, $25 \%$ w/v PEG 3,350), with $1 \mathrm{mM}$ methyl AMP and $1 \mathrm{mM}$ TCEP. $15 \%(\mathrm{v} / \mathrm{v})$ ethylene glycol was used as a cryoprotectant. Crystals containing methyl AMP and coenzyme A were grown in sparse matrix screen MCSG1 condition a6 (18.71\% PEG 3,350, 0.2M Lithium acetate), with $1 \mathrm{mM}$ methyl AMP, $1 \mathrm{mM}$ TCEP, and $1 \mathrm{mM}$ coenzyme A. $20 \%(\mathrm{v} / \mathrm{v})$ ethylene glycol was used as a cryoprotectant. Ethyl AMP containing crystals were grown in optimization conditions based on screen MCSG1 condition h3 (200mM lithium acetate, 18.32-19.1\% PEG 3350), with $1 \mathrm{mM}$ ethyl-AMP and $1 \mathrm{mM}$ TCEP. Crystals containing a complex with ethyl AMP and coenzyme A were setup in similar conditions with the addition of $1 \mathrm{mM}$ coenzyme A. $20 \%(\mathrm{v} / \mathrm{v})$ ethylene glycol was used as a cryoprotectant. Finally, crystals containing propyl AMP were grown in sparse matrix screen MCSG1 condition h5 (200 mM Potassium chloride, 20\% (w/v) PEG 3350), with $1 \mathrm{mM}$ pro-pyl AMP and $1 \mathrm{mM}$ TCEP. $20 \%(\mathrm{v} / \mathrm{v})$ ethylene glycol was used as a cryoprotectant.

\section{Cryptococcus neoformans}

Crystals of Cryptococcus neoformans ACS1 were generally grown at $10 \mathrm{mg} / \mathrm{ml}$ in optimization conditions based on screen Wizard Classic 1\&2 (Rigaku Reagents) condition e8 (10-20\% (w/v) PEG 8000, 0.1M Na/K phosphate, $\mathrm{pH} 5.8-7)$. 20\% (v/v) ethylene glycol was used as a cryoprotectant. Where applicable, AMP esters were added at 1mM (propyl, ethyl, and butyl AMP), coen-zyme A at $1-2 \mathrm{mM}$, acetyl AMS at $0.5 \mathrm{mM}, \mathrm{ATP} / \mathrm{MgCl} 2$ at $1 \mathrm{mM}$ and TCEP at $1 \mathrm{mM}$. Crystals of apo/acetylated active site lysine 640 from Cryptococcus neoformans were grown in sparse matrix screen Morpheus (Rigaku Reagents) condition h3 (10.0\% w/v PEG4,000, 20\% glycerol, 0.02M amino acid mix, 0.1M MES/imidazole pH6.5) (34). No additional cryo-protectant was used.

\section{Structure determination}


The structures of the fungal ACS1 apo or with compound were collected between 2016-2020 at the Advanced Photon Source, beamlines 21-ID-F and G (CCD Rayonix MX-225 and MX-300 Xray detector respectively), with the exception of 7KDS (Candida albicans ACS2) which was collected at the Canadian Lightsource (Pilatus3 S 6M X-ray detector). Data were reduced with

XDS/XSCALE, (3) and solved by molecular replacement using Phaser with input models based upon previously solved crystal structures of fungal, yeast or bacterial ACS1. Iterative manual model building using Coot (4) and Phenix.Refine continued until R and Rfree converged (5). Model quality was validated using Coot and MolProbity (6). Structures were validated using Molprobity prior to deposition in the Protein Data Bank (See Crystallographic Table) $(7,8)$. Diffraction images are available on Integrated Resource for Reproducibility in Macromolecular Crystallography (http://www.proteindiffraction.org) (9-11).

\section{References:}

1. Qiao, C.; Gupte, A.; Boshoff, H. I.; Wilson, D. J.; Bennett, E. M.; Somu, R. V.; Barry, C. E., 3rd; Aldrich, C. C., 5'-O-[(N-acyl)sulfamoyl]adenosines as antitubercular agents that inhibit MbtA: an adenylation enzyme required for siderophore biosynthesis of the mycobactins. J Med Chem 2007, 50 (24), 6080-94.

2. Miller KD, Pniewski K, Perry CE, Papp SB, Shaffer JD, Velasco-Silva JN, Casciano JC, Aramburu TM, Srikanth YVV, Cassel J, Skordalakes E, Kossenkov AV, Salvino JM, Schug ZT. (2021) Targeting ACSS2 with a transition-state mimetic inhibits triple-negative breast cancer growth. Cancer Res. 81, 1252-1264.

3. Gorrec F. The MORPHEUS protein crystallization screen. J Appl Crystallogr. 2009 Dec 1;42(Pt 6):1035-1042. doi: 10.1107/S0021889809042022. Epub 2009 Nov 7. PMID: 22477774 ; PMCID: PMC3246824.

4. Kabsch, W. (2010). Xds. Acta Crystallographica Section D: Biological Crystallography $66,125-132$.

5. Emsley, P., Lohkamp, B., Scott, W.G. and Cowtan, K. (2010) Features and development of Coot. Acta Crystallographica Section D: Biological Crystallography 66, 486-501.

6. Adams, P.D., Afonine, P.V., Bunkóczi, G., Chen, V.B., Davis, I.W., Echols, N., Headd, J.J., Hung, L.W., Kapral, G.J., Grosse-Kunstleve, R.W. and McCoy, A.J. (2010) PHENIX: a com-prehensive Python-based system for macromolecular structure solution. Acta Crystallograph-ica Section D: Biological Crystallography 66, 213-221.

7. Chen, V.B., Arendall, W.B., Headd, J.J., Keedy, D.A., Immormino, R.M., Kapral, G.J., Murray, L.W., Richardson, J.S. and Richardson, D.C. (2010) MolProbity: all-atom structure 
validation for macromolecular crystallography. Acta Crystallographica Section D: Biological Crystallog-raphy 66, 12-21.

8. Berman, H., Henrick, K. and Nakamura, H. (2003) Announcing the worldwide protein data bank. Nature Structural \& Molecular Biology, 10, 980-980.

9. Berman, H.M., Westbrook, J., Feng, Z., Gilliland, G., Bhat, T.N., Weissig, H., Shindyalov, I.N. and Bourne, P.E. (2000) The protein data bank. Nucleic Acids Research 28, 235-242.

10. Grabowski M, Langner KM, Cymborowski M, Porebski PJ, Sroka P, Zheng H, Cooper DR, Zimmerman MD, Elsliger MA, Burley SK, Minor W (2016) A public database of macromolecu-lar diffraction experiments. Acta crystallographica. Section D, Structural biology 72 (Pt 11):1181-1193.

11. Grabowski M, Cymborowski M, Porebski PJ, Osinski T, Shabalin IG, Cooper DR, Minor W (2019) The Integrated Resource for Reproducibility in Macromolecular Crystallography: Expe-riences of the first four years. Struct Dyn 6: 064301. 